\author{
Universidade de São Paulo \\ Instituto de Astronomia, Geofísica e Ciências Atmosféricas \\ Departamento de Ciências Atmosféricas
}

André Seiji Wakate Teruya

\title{
Nonlinear Interaction of Gravity and Acoustic Waves
}

(Interações Não-Lineares entre

Ondas de Gravidade e Acústicas)

São Paulo 

André Seiji Wakate Teruya

\title{
Nonlinear Interaction of Gravity and Acoustic Waves
}

\author{
(Interações Não-Lineares entre \\ Ondas de Gravidade e Acústicas)
}

\author{
Tese apresentada ao Departamento de \\ Ciências Atmosféricas do Instituto de \\ Astronomia, Geofísica e Ciências \\ Atmosféricas da Universidade de São Paulo \\ como requisito parcial para a obtenção \\ do título de Doutor em Ciências. \\ Área de Concentração: Meteorologia \\ Orientador: Prof. Dr. Carlos Frederico \\ Mendonça Raupp
}

São Paulo 

To my beloved parents. / Aos meus amados pais. 



\section{Acknowledgements}

Aos meu pais Roberto e Lenir e ao meu irmão Key, pelo amor, carinho e apoio ao longo da minha vida;

À Kaori, pelo suporte e amor concedidos nestes últimos dez anos;

Ao meu orientador, Prof. Dr. Carlos Frederico Mendonça Raupp, pela oportunidade de trabalhar com um tema encantador, por sua paciência, amizade e imprescindível orientação acadêmica nesses quase dez anos de trabalho em equipe.

Aos amigos e colegas do Instituto de Física (IF), Instituto de Matemática e Estatística (IME) e do Instituto de Astronomia, Geofísica e Ciências Atmosféricas (IAG);

Agradeço à CAPES e CNPq pelo financiamento da minha pesquisa.

This thesis was written in $\mathrm{AT}_{\mathrm{EX}}$ with the class IAGTESE, developed to write thesis and dissertations from IAG. 



\section{Resumo}

Neste trabalho investigou-se a possibilidade de ondas acústicas serem instáveis em relação à perturbações nos modos gravidade-inerciais, através de interações triádicas nãolineares no contexto do modelos não-hidrosatático raso. Foi considerado uma expansão de Galerkin, altamente truncada, das perturbações em torno de um campo básico isotérmico, hidrostático e em repouso. A expansão de Galerkin foi feita em relação às autofunções do problema linearizado. Para um tripleto isolado, ficou demonstrado que uma onda acústica não consegue amplificar as perturbações de um par de ondas gravidade-inerciais. Isso ocorre devido ao alto mismatch das autofrequências do tripleto de ondas. Por outro lado, a análise da dinâmica de dois tripletos acoplados por um único modo demonstrou que um modo gravidade-inercial não-hidrostático (acústico), participando de um tripleto quaseressonate com dois modos acústicos (um modo gravidade-inercial e um modo acústico) pode ser instável em relação à pequenas perturbações associadas a um par de ondas gravidadeinerciais que estão em balanço hidrostático. Esta instabilidade linear implica em uma troca de energia inter-triádica significativa, se a não-linearidade associada ao segundo tripleto, composto por dois modos gravidade-inearciais hidrostáticos, for reconsiderada. As implicações desses resultados para o ajuste hidrostático não-linear são discutidas. 



\section{Abstract}

Here we have investigated the possibility of an inertio-acoustic wave-mode to be unstable with regard to gravity mode perturbations through nonlinear triad interactions in the context of a shallow nonhydrostatic model. We have considered highly truncated Galerkin expansions of the perturbations around a resting, hydrostatic and isothermal background state in terms of the eigensolutions of the linear problem. For a single interacting wave triplet, we have shown that an acoustic mode cannot amplify a pair of inertio-gravity perturbations due to the high mismatch among the eigenfrequencies of the three interacting wave-modes, which resquires an unrealistically high amplitude of the acoustic mode in order for pump wave instability to occur. In contrast, it has been demonstrated by analyzing the dynamics of two triads coupled by a single mode that a non-hydrostatic gravity wave-mode participating of a nearly resonant interaction with two acoustic modes can be unstable to small amplitude perturbations associated with a pair of two hydrostatically balanced inertio-gravity wave-modes. This linear instability yields significant inter-triad energy exchanges if the nonlinearity associated with the second triplet containing the two hydrostatically balanced inertio-gravity modes is restored. Therefore, this inter-triad energy exchanges lead the acoustic modes to yield significant energy modulations in hydrostatic inertio-gravity wave modes. The implications of the results for the nonlinear hydrostatic adjustment problem are discussed. 



\section{List of Figures}

3.1 Dispersion curves of inertia-acoustic (IA) and inertia-gravity (IG) waves corresponding to the first three baroclinic modes $k=1,2,3$. All the curves are referred to the meridional index $n=1$. In this figure $j$ is the zonal wave index, $m$ is the vertical wave index. . . . . . . . . . . . . . . . 4

3.2 Similar to Fig. 3.1, but only for the inertia-gravity waves (IG) and their correspondent dispersion curves obtained by hydrostatic approximation (H). 48

3.3 Equivalent height of acoustic (AI) and gravity modes (IG). In this figure $j$ is the zonal wave index, $m$ is the vertical wave index. All curves are for meridional index $1 . \ldots \ldots \ldots$. . . . . . . . . . . 49

3.4 Gravity modes energy partition for vertical mode $k_{i}=1$ and meridional index $n_{i}=1$.

3.5 Gravity modes energy partition for vertical mode $k_{i}=5$ and meridional index $n_{i}=1$.

3.6 Gravity modes energy partition for vertical mode $k_{i}=1$ and meridional index $n_{i}=5$.

3.7 Acoustic modes energy partition for vertical mode $k_{i}=1$ and meridional

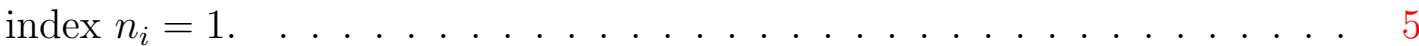

3.8 Acoustic modes energy partition for vertical mode $k_{i}=5$ and meridional index $n_{i}=1$

3.9 Acoustic modes energy partition for vertical mode $k_{i}=1$ and meridional index $n_{i}=100$.

4.1 Time evolution of the energy of a resonant triad (triad 1 from Table 4.1).

4.2 Time evolution of the energy of a nonresonant triad (triad 14 from Table 4.1). 65 
4.3 Numerical solution of the duet system forced by triad 1 and composed by modes $(170,1,2, \mathrm{IG}),(169,1,2, \mathrm{IG})$ from triad 2. This solution presented a maximal Lyapunov exponent $\lambda_{L}=1.35 E-5$. . . . . . . . . . . .

4.4 Numerical solution of the duet system forced by triad 1 and composed by modes $(170,1,1, \mathrm{IG}),(169,1,1, \mathrm{IG})$ from triad 13. This solution presented a maximal Lyapunov exponent $\lambda_{L}=-4.85 E-10 \ldots \ldots$. . . . . . . . 68

4.5 Numerical solution of the duet system forced by triad 1 and composed by modes $(169,1,2, \mathrm{IG}),(-168,1,1, \mathrm{IG})$ from triad 15. This solution presented a maximal Lyapunov exponent $\lambda_{L}=6.59 E-11 \ldots$. . . . . . . . . . . . 69

4.6 Numerical solution of a quintet composed of triads 1 and 2 from table 4.1. 70

4.7 Numerical solution of a quintet composed of triads 1 and 2 from table 4.1. 71 


\section{List of Tables}

4.1 Each mode is represented by a zonal wavenumber, meridional wavenumber, vertical wavenumber and kind of wave. Frequencies $\omega$ and mismatch $\delta_{a b c}$ are measured in Hertz. Modes 1,2,4,9,10 and 11 are nearly resonant. . . . . 70 



\section{Contents}

1. Introduction . . . . . . . . . . . . . . . . . . . 17

2. Hamiltonian Formalism . . . . . . . . . . . . . . . . . . . . . 23

2.1 Finite dimensional Hamiltonian systems . . . . . . . . . . . . . . . . 23

2.2 Infinite Dimensional Hamiltonian Systems . . . . . . . . . . . . . . . . 27

2.3 Chapter Summary . . . . . . . . . . . . . . . . . . . . . . . . 31

3. Governing Equations and Normal Modes . . . . . . . . . . . . . . . . . . 33

3.1 Governing Equations - Linear Problem . . . . . . . . . . . . . . . 39

3.2 Separation of Variables . . . . . . . . . . . . . . . . . . . . . 41

3.3 Normal Modes on a $f$-plane. . . . . . . . . . . . . . . . . . . . . . 44

3.3.1 Eigenfunctions Meridional and Vertical Structures . . . . . . . . . . 44

3.3.2 Eigenfunctions Orthogonality and Completeness . . . . . . . . . . 45

3.4 Linear Dispersion . . . . . . . . . . . . . . . . . . . . . . 46

3.5 Normal Modes Energetics . . . . . . . . . . . . . . . . 46

3.5.1 Gravity Modes ..................... 46

3.5.2 Acoustic Modes . . . . . . . . . . . . . . . . . . 47

4. Weakly Nonlinear Dynamics . . . . . . . . . . . . . . . . . . . . . . . 57

4.1 Modal expansion . . . . . . . . . . . . . . . . . 57

4.2 Energy constraints for the interacting triads . . . . . . . . . . . . 59

4.3 Analysis of Highly Truncated Spectral Solutions . . . . . . . . . . . . . . . 61

4.3.1 Single triad interaction . . . . . . . . . . . . . . . . . . 61

4.3.2 Two triads coupled by one mode . . . . . . . . . . . . . . . 64 
5. Conclusions . . . . . . . . . . . . . . . . . . . . 73

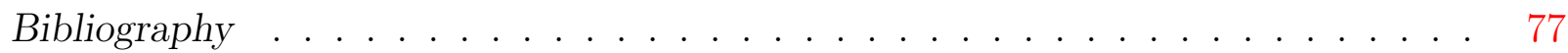


Chapter 1

\section{Introduction}

With the advent of high resolution atmospheric models, there has been a renewed interest in the study of the normal modes of nonhydrostatic atmospheric models. Kasahara and Qian (2000) and Qian and Kasahara (2003) studied the linear normal mode function theory of the shallow nonhydrostatic model (White et al., 2005) in the contexts of spherical and the beta-plane geometries, respectively. Their theory has been augmented with the account of the nontraditional Coriolis terms (Kasahara, 2003b,a). The linear normal mode function theory has also been presented by Kasahara (2004) for the full deep nonhydrostatic case, as well as by Kasahara and Gary (2006) for the Boussinesq system in which acoustic modes are absent. Nevertheless, as the governing equations of the atmospheric dynamics are nonlinear, a more accurate account of the normal mode theory should also include the effect of the nonlinearity on the waves' dynamics.

Atmospheric normal mode function theory has long received considerable research effort due to the central role of normal modes in theoretical toy models. These models are designed to elucidate mechanisms of the atmospheric dynamics that are not distinguishable in a full general circulation models (AGCMs). Also, there are practical applications of normal modes in data assimilation (Žagar et al., 2004) and initialization of numerical weather prediction models (Baer and Tribbia, 1977; Machenhauer, 1977; Leith, 1980). A historical account of research on normal modes of atmospheres over spheres can be found in Kasahara (2004). In addition, the normal mode functions of three-dimensional primitive equations in spherical coordinates can be utilized in diagnostic analysis of AGCM's outputs by projecting global circulation dynamical fields onto these eigenfunctions (see, for instance, Kasahara and Puri, 1981; Žagar et al., 2015).

The normal modes of a dynamical system represent its free small amplitude oscillati- 
ons around a reference steady-state. They are the eigensolutions of the linearized version of the correspondent partial differential equations. For the atmosphere, this reference steady-state is usually defined as with no motion and characterized by a stably stratified atmosphere, since in this case the leading-order wave actions by which the orthogonality relations hold, are positive definite integrals, represented by either pseudoenergy or pseudomomentum (see, Shepherd, 1990; Vallis, 2006), and consequently the eigensolutions represent purely stable wave motions. In this context, the effect of nonlinearity is to break down the independence of the normal modes, allowing their energy to be exchanged within triads of resonant eigenmodes (see Raupp et al., 2008, and references therein).

Due to the extensive use of global numerical models with coarse resolutions until the nineties, the research effort on atmospheric normal mode functions has mostly focused on global hydrostatic primitive equation models (Longuet-Higgins, 1968; Kasahara, 1976, 1977; Kasahara, 1978), as well as their equatorial beta-plane (Matsuno, 1966; Lindzen, 1967; Silva Dias et al., 1983; DeMaria, 1985; Raupp et al., 2008; Raupp and Silva Dias, 2009) and quasi-geostrophic (Vallis 2006; Pedlosky 1987 and references therein) approximations. Nevertheless, with the increasing utilization of high-resolution numerical models during the last decades, the interest in normal mode function theory of global non-hydrostatic models has been renewed. Kasahara and Qian (2000) studied the linear theory of non-hydrostatic normal modes of a shallow global non-hydrostatic model in spherical coordinates, while Qian and Kasahara (2003) performed a similar analysis for the equatorial and mid-latitude beta-planes. The shallow non-hydrostatic model adopted in these studies considers the traditional approximation (Phillips, 1973) and neglects all the apparent acceleration terms involving the vertical velocity in the horizontal momentum equations, as well as all the apparent acceleration terms of the vertical momentum equation.

In this context, Kasahara (2003b,a) augmented the linear theory of non-hydrostatic normal modes developed in Kasahara and Qian (2000) and Qian and Kasahara (2003) by accounting for the nontraditional Coriolis terms proportional to the cosine of latitude in the momentum equations in the context of the tangent plane geometry. He analyzed the role of these nontraditional terms in the dispersion relation of inertio-gravity (IG) and inertio-acoustic (IA) modes. Kasahara (2004) computed the normal modes of the full deep non-hydrostatic global model, including all the curvature and nontraditional Coriolis terms of the momentum conservation in spherical coordinates, along with not performing 
the traditional approximation. Unlike the hydrostatic primitive equations and the shallow non-hydrostatic model, in the deep non-hydrostatic model in spherical coordinates, the linearized equations around a resting basic state are no longer separable into two simultaneous eigenvalue problems representing the vertical and horizontal structures of the normal modes. Therefore, Kasahara (2004) proposed a numerical procedure involving a finitedifference discretization in the vertical direction and the meridional eigenvalue problem is solved for each vertical level. He then compared the solutions of the deep non-hydrostatic model with those of the shallow non-hydrostatic model obtained by Kasahara and Qian (2000).

Recently, Teruya (2014) extended the work of Kasahara and Qian (2000) by analyzing both linear and weakly nonlinear energetics of inertia-gravity and inertia-acoustic modes. The former analyzed the dynamics of a single resonant triad interaction involving an inertia-gravity wave and two inertia-acoustic modes. In this kind of resonant interaction, an inertia-gravity wave acts as a catalyst mode for the energy exchanges between the two inertia-acoustic waves. So the amplitude of the inertia-gravity wave is quasi-stationary, but it enables the interaction between the two inertia-acoustic waves and controls both the interaction period and the impacts of the energy modulations on the perturbed dynamical field variables. Therefore, comparing this finding with previous investigations on the nonlinear atmospheric wave theory in the hydrostatic context (e.g., Vanneste and Vial, 1994; Raupp et al., 2008; Ripa, 1983a,b; Domaracki and Loesch, 1977; Loesch and Deininger, 1979; Duffy, 1974), it is clear that the role of an inertia-gravity mode in a resonant interaction involving inertia-acoustic modes is similar to the role of a Rossby mode in a resonant interaction involving two inertio-gravity waves.

This thesis is a follow up of the work of Teruya (2014) by further investigating the nonlinear dynamics of the shallow nonhydrostatic equations. In particular, we are interested here in investigating the possibility of a resonant triad involving inertia-acoustic and nonhydrostatic inertia-gravity waves to be unstable with regard to interacting triads (not necessarily resonant) of inertia-gravity modes. A motivation for this analysis stems from recent findings in the nonlinear wave literature pointing out that even though in a single interacting wave triad the resonance relation among the modes' eigenfrequencies is crucial for significant energy exchanges to occur in the limit of weak nonlinearity. If one relaxes the resonance assumption of weak nonlinearity to take into account off-resonant wave tri- 
ads, the mismatch among the wave frequencies within interacting triads might be relevant for the energy flow throughout the whole modal space. Bustamante et al. (2014) showed in a reduced dynamical system of a resonant triad (RT) and a nonresonant (NRT) triad coupled by two modes (i.e., a four-wave system) that, for moderate values of wave amplitudes, the energy leakage from the RT toward the NRT increases as the nonlinear frequency of amplitude (energy) modulations of the RT approaches the frequency mismatch among the wave eigenfrequencies of the NRT. The authors have labeled this synchronization between the nonlinear frequency and linear mismatch frequency between different interacting triads as precession resonance. Bustamante et al. (2014) also demonstrated the critical role of precession resonance mechanism for increasing the efficiency of the energy flow throughout the whole system of several connected triads. Another mechanism that yields significant energy transfers throughout the whole modal space in a diversity of wave problems is the modulational instability (Connaughton et al., 2010). Thuburn (2011) points out that internal acoustic waves and internal inertio-gravity waves are energetically weak, and are relatively fast processes compared to the energetically dominant processes observed in the atmosphere. Furthermore, since these waves impose a huge constraint in the time step in explicit time schemes Pielke (2002), one might want to damp/distort their propagation with semi-implicit time schemes to avoid the time step constraint present in explicit time schemes (Giraldo et al., 2010). There are other ways to avoid the time step constraint of explicit time schemes, for example, Daley (1988) proposed a filter for acoustic modes based on normal mode expansion, in the same spirit of the method proposed by Tribbia (1979) in the hydrostatic context to filter out inertio-gravity waves, using split-explicit time schemes (Klemp et al., 2007; Klemp et al., 2018), using approximations to filter out acoustic modes (Davies et al., 2003; Klein, 2009).

According to Thuburn (2011):

"Atmospheric dynamics is complex and involves a wide range of space and time scales. The energetically dominant dynamics is slow and close to balance, and it may be wavelike, vortical, or strongly nonlinear. Fast acoustic and inertiogravity waves represent departures from balance, but are also the mechanism by which the atmosphere continuously adjusts towards balance. Nonlinearity implies interac- tions between the different space and time scales. Particularly important are energy and potential enstrophy transfers across scales; for any 
practical global atmospheric model there will inevitably be important dynamics occurring near the resolution limit. The need to capture all of these processes with sufficient accuracy make numerical modeling of the atmosphere one of the most challenging branches of computational fluid dynamics."

So, it is crucial to understand the role of acoustic and inertia-gravity waves with a linear and nonlinear perspective.

Our analysis of a highly truncated spectral model of the shallow nonhydrostatic equations demonstrates that an inertia-gravity mode, participating of a resonant triad interaction with two inertia-acoustic modes, can be unstable to small amplitude perturbations if that inertia-gravity mode is coupled with two lower frequency inertio-gravity modes. Since the high frequency inertio-gravity wave is more nonhydrostatic than low frenquency inertiogravity waves, our results suggest that ultra-high frequency acoustic modes can potentially yield amplitude (energy) modulations in hydrostatically balanced inertio-gravity waves through inter-triad energy exchanges. Therefore, the present theoretical description suggests that acoustic modes excited by localized and explosive heating associated with convective storms might play an important role in both hydrostatic and geostrophic adjustments, as will be discussed in Chapter 5 .

This thesis organized as follows. In Chapter 2 we review the Hamiltonian formalism for fluids. Then, in Chapter 3 we use the Hamiltonian formalism to derive the equations of motion and the integrals of motion of the nonhydrostatic shallow model. The spectral representation of the solution in terms of the eigenmodes of the linearized problem is also presented in the latter chapter. In Chapter 4 we present the general properties of the interaction coefficients in an arbitrary interacting wave triad as a consequence of total pseudo-energy conservation and the reduced dynamics of one wave triad and two triads coupled by a single mode to investigate the possibility of acoustic modes to excite hydrostatic inertio-gravity waves. The main conclusions are discussed in Section 5. 
Chapter 2

\section{Hamiltonian Formalism}

One of the main advantages of using the Hamiltonian formalism is that it provides a systematic procedure to find out the integrals of motion of a dynamical system (Salmon, 1998). It is also possible to figure out which integrals of motion as kept after applying some approximation on a model (Shepherd, 1990; Morrison, 1998). A handful of geophysical fluid dynamics models can be described using the Hamiltonian formalism (Shepherd, 1990; Scinocca and Shepherd, 1992; Ripa et al., 2003). Often, to characterize the motion of a geophysical fluid, one should use the Hamiltonian formalism in its symplectic representation (Shepherd, 1990). A few applications of the Hamiltonian formalism will be worked out in the next section. These examples will be used to define some mathematical notations and to illustrate the advantages of using the Hamiltonian formalism in finite and infinite dimension systems.

\subsection{Finite dimensional Hamiltonian systems}

A $N$-dimensional canonical Hamiltonian system is defined by a phase space $\left\{\left(q_{i}, p_{i}\right)\right\}_{i=1, \ldots, N}$, where $q_{i}$ 's are generalized coordinates and $p_{i}$ 's are generalized momentum, and a Hamiltonian function $H\left(q_{i}, p_{i}, t\right)$. The canonical Hamilton equations fully describe the system's dynamical evolution (Landau and Lifshitz, 1976; Goldstein, 1980)

$$
\begin{aligned}
\frac{d q_{i}}{d t} & =\frac{\partial H}{\partial p_{i}} \\
\frac{d p_{i}}{d t} & =-\frac{\partial H}{\partial q_{i}} .
\end{aligned}
$$

In the canonical representation, every integral of motion is associated with a symmetry in the Hamiltonian function. For example, if the Hamiltonian is time-independent (i.e., 
$\partial H / \partial t=0)$, then

$$
\begin{aligned}
\frac{d H}{d t} & =\frac{\partial H}{\partial q_{i}} \frac{d q_{i}}{d t}+\frac{\partial H}{\partial p_{i}} \frac{d p_{i}}{d t} \\
& =\left(-\frac{d p_{i}}{d t}\right) \frac{d q_{i}}{d t}+\left(\frac{d q_{i}}{d t}\right) \frac{d p_{i}}{d t} \\
& =0
\end{aligned}
$$

Thus, if the Hamiltonian function has a translational symmetry in time, then it is a integral of motion of the system.

Example Consider an object with mass $m$ attached to a massless spring with spring constant $k$ (i.e. a harmonic oscillator). The Lagrangian $L=L(x, \dot{x})$ of the system is $L=K-V$, where $K$ is the kinetic energy and $V$ is the potential energy of the system (Landau and Lifshitz, 1976). Thus, the Lagrangian of the harmonic oscillator is

$$
L(x, \dot{x})=\frac{m \dot{x}^{2}}{2}-\frac{k x^{2}}{2}
$$

The Hamiltonian functions is $H=H(x, p)$, where $p \equiv \partial L / \partial \dot{x}=m \dot{x}$ is the generalized momentum associated to the generalized coordinate $x$. The Hamiltonian function of the system can be obtained using the Legendre transform (Landau and Lifshitz, 1976),

$$
\begin{aligned}
H(x, p) & =p \dot{x}-L(x, \dot{x}) \\
& =p \dot{x}-\left(\frac{p^{2}}{2 m}-\frac{k x^{2}}{2}\right) \\
& =\frac{p^{2}}{2 m}+\frac{k x^{2}}{2} .
\end{aligned}
$$

Since the Hamiltonian function is time-independent, then, without glancing at the equations of motion, we know that $H$ is an integral of motion of the system. Using Hamilton's equations gives us the time evolution equation's

$$
\begin{aligned}
& \frac{\partial H}{\partial q}=\dot{p} \Rightarrow \frac{\partial H}{\partial x}=k x=\dot{p} \\
& \frac{\partial H}{\partial p}=\dot{q} \Rightarrow \frac{H}{\partial p}=\dot{x}=\frac{p}{m}
\end{aligned}
$$

eliminating $p$,

$$
\ddot{x}=\frac{k}{m} x
$$

which is the well-known harmonic oscillator equation. 
Hamilton's equations can also be cast using the symplectic notation Hamiltoniano(Shepherd, 1990; Goldstein, 1980)

$$
\frac{d u_{i}}{d t}=J_{i j} \frac{\partial H(\boldsymbol{u})}{\partial u_{j}}
$$

where $u$ is the state vector of the dynamical system, $J_{i j}$ is a skew-symmetric tensor that also satisfies the Jacobi's identity

$$
\epsilon_{i j k} J_{i m} \frac{\partial J_{j k}}{\partial u_{m}}=0
$$

where $\epsilon_{i j k}$ is the Levi-Civita symbol.

For the canonical representation we have,

$$
\begin{aligned}
\boldsymbol{u} & =\left(q_{1}, \ldots, q_{N}, p_{1}, \ldots, p_{N}\right)^{T} \\
\boldsymbol{J} & =\left(\begin{array}{cc}
0_{N} & I_{N} \\
-I_{N} & 0_{N}
\end{array}\right)
\end{aligned}
$$

where $0_{N}$ a $N$ by $N$ zero matrix and $I_{N}$ is the $N$ by $N$ identity matrix.

The symplectic notation can also be written in terms of a Poisson bracket $[\cdot, \cdot]$. The Poisson bracket is defined as

$$
[F, G]=\frac{\partial F}{\partial u_{i}} J_{i j} \frac{\partial G}{\partial u_{j}},
$$

where $F(\boldsymbol{u})$ and $G(\boldsymbol{u})$ are state functions. The Poisson bracket maps two state functions to another state function. It is also a bilinear, skew-symmetric mapping. The canonical Poisson brackets are,

$$
[F, G]=\frac{\partial F}{\partial q_{i}} \frac{\partial G}{\partial p_{i}}-\frac{\partial F}{\partial p_{i}} \frac{\partial G}{\partial q_{i}} .
$$

With the bracket notation, the equations of motions are written as

$$
\begin{aligned}
\frac{d F}{d t} & =\frac{\partial F}{\partial q_{i}} \frac{d q_{i}}{d t}+\frac{\partial F}{\partial p_{i}} \frac{d p_{i}}{d t} \\
& =\frac{\partial F}{\partial q_{i}} \frac{\partial H}{\partial p_{i}}+\frac{\partial F}{\partial p_{i}}\left(-\frac{\partial H}{\partial q_{i}}\right) \\
& =[F, H] .
\end{aligned}
$$

Also, the Jacobi identity in bracket notation is

$$
[F,[G, H]]+[H,[F, G]]+[G,[H, F]]=0 .
$$


It is trivial, yet pedestrian, to show that the Canonical Poisson brackets satisfy the Jacobi identity. Notice that $\operatorname{ker}[J]=\mathbf{0}$.

The noncanonical Hamiltonian formalism relies on the fact that one can define a bracket different from the canonical Poisson bracket, but that is also bilinear, skew-symmetric, and satisfy the Jacobi identity. The main difference is that in the noncanonical case $\operatorname{ker}[J] \neq \mathbf{0}$. This property results in a new class of conservative quantities, the Casimir invariant. A state function $C$ that commutes with any state function is called a Casimir function

$$
[C, G]=0 \text { for any } G=G(\boldsymbol{u})
$$

Notice that the commutator of the Casimir with the Hamiltonian is

$$
[C, H]=-[H, C]=\frac{\partial C}{\partial u_{i}} J_{i j} \frac{\partial H}{\partial u_{j}}=-\frac{\partial H}{\partial u_{i}} J_{i j} \frac{\partial C}{\partial u_{j}}=0 \Leftrightarrow J_{i j} \frac{\partial C}{\partial u_{j}}=0
$$

Example The motion of a rigid body is described by the Euler's rigid body equations (Goldstein, 1980; Landau and Lifshitz, 1976)

$$
\begin{aligned}
& \dot{\Pi}_{1}=\frac{I_{2}-I_{3}}{I_{2} I_{3}} \Pi_{2} \Pi_{3} \\
& \dot{\Pi}_{2}=\frac{I_{3}-I_{1}}{I_{3} I_{1}} \Pi_{3} \Pi_{1} \\
& \dot{\Pi}_{3}=\frac{I_{1}-I_{2}}{I_{1} I_{2}} \Pi_{1} \Pi_{2}
\end{aligned}
$$

where $\Pi_{i}=I_{i} \Omega_{i}$ are the angular momentum associated with the body's principal axes of inertia and $I_{i}$ 's are the inertia moments. Notice that the rigid body's motion is described by just three differential equations. With the canonical formalism the state vector must be $\boldsymbol{u}=\left(x, y, z, \phi, \theta, \psi ; p_{x}, p_{y}, p_{z}, p_{\phi}, p_{\theta}, p_{\psi}\right)^{T}$. Where $x, y$ e $z$ are cartesian coordinates, $\phi$, $\theta$ e $\psi$ are the Euler angles and $p_{x}, p_{y}, p_{z}, p_{\phi}, p_{\theta}, p_{\psi}$ are their respective momentum. So, the canonical Hamiltonian formalism we will end up with a set of six differential equations.

In the noncanonical formalism (Shepherd, 1990), we have

$$
\begin{aligned}
\boldsymbol{u} & =\left(\Pi_{1}, \Pi_{2}, \Pi_{3}\right)^{T} \\
H(\boldsymbol{\Pi}) & =\frac{1}{2}\left(\frac{\Pi_{1}^{2}}{I_{1}}+\frac{\Pi_{2}^{2}}{I_{2}}+\frac{\Pi_{1}^{3}}{I_{3}}\right) \\
\boldsymbol{J} & =\left(\begin{array}{ccc}
0 & -\Pi_{3} & \Pi_{2} \\
\Pi_{3} & 0 & -\Pi_{1} \\
-\Pi_{2} & \Pi_{1} & 0
\end{array}\right)
\end{aligned}
$$


The rigid body noncanonical Poisson bracket is (Marsden and Ratiu, 1999)

$$
[F, G](\Pi)=-\Pi \cdot(\nabla F \times \nabla G)
$$

where $\nabla=\left(\partial_{\Pi_{1}}, \partial_{\Pi_{2}}, \partial_{\Pi_{3}}\right)$. Explicitly, the noncanonical Poisson bracket yields,

$$
[F, H]=-\left(\Pi_{1}, \Pi_{2}, \Pi_{3}\right) \cdot\left(\frac{\Pi_{3}}{I_{3}} \frac{\partial F}{\partial \Pi_{2}}-\frac{\Pi_{2}}{I_{2}} \frac{\partial F}{\partial \Pi_{3}},-\frac{\Pi_{3}}{I_{3}} \frac{\partial F}{\partial \Pi_{1}}+\frac{\Pi_{1}}{I_{1}} \frac{\partial F}{\partial \Pi_{3}}, \frac{\Pi_{2}}{I_{2}} \frac{\partial F}{\partial \Pi_{1}}-\frac{\Pi_{1}}{I_{1}} \frac{\partial F}{\partial \Pi_{2}}\right) .
$$

Taking $F=\Pi_{1}, F=\Pi_{2}$ and $F=\Pi_{3}$ results in

$$
\begin{aligned}
& {\left[\Pi_{1}, H\right]=-\frac{\Pi_{2} \Pi_{3}}{I_{2} I_{3}}\left(I_{3}-I_{2}\right)=\dot{\Pi}_{1}} \\
& {\left[\Pi_{2}, H\right]=-\frac{\Pi_{1} \Pi_{3}}{I_{1} I_{3}}\left(I_{1}-I_{3}\right)=\dot{\Pi}_{2}} \\
& {\left[\Pi_{3}, H\right]=-\frac{\Pi_{1} \Pi_{2}}{I_{1} I_{2}}\left(I_{2}-I_{1}\right)=\dot{\Pi}_{3}}
\end{aligned}
$$

which are exactly the Euler rigid body equations. Notice that the total angular momentum is a Casimir function of the system $C=1 / 2\left(\Pi_{1}^{2}+\Pi_{2}^{2}+\Pi_{3}^{2}\right)$. Since $\nabla C=\left(\Pi_{1}, \Pi_{2}, \Pi_{3}\right)=\Pi$, then

$$
\begin{aligned}
\frac{d C}{d t} & =[C, F] \\
& =-\boldsymbol{\Pi} \cdot(\nabla C \times \nabla F) \\
& =-\boldsymbol{\Pi} \cdot(\boldsymbol{\Pi} \times \nabla F) \\
& =0
\end{aligned}
$$

Thus $C$ commutes with any state function $F$. In particular, if we take $F=H$, it is clear that the Casimir function $C$ is also an integral of motion

\subsection{Infinite Dimensional Hamiltonian Systems}

Some fluid dynamics models can be described using the Hamiltonian formalism (Shepherd, 1990; Scinocca and Shepherd, 1992; Ripa et al., 2003). For fluids, the symplectic representation is the same as in (2.7), but instead of partial derivatives, we must use variational/functional derivatives,

$$
\boldsymbol{u}_{t}=\boldsymbol{J} \frac{\delta \mathscr{H}}{\delta \boldsymbol{u}}
$$


where $\boldsymbol{u}$ is a column vector with a function in each entry, $\boldsymbol{u}_{t}$ is the element-wise time derivative of $\boldsymbol{u}, \mathscr{H}$ is the Hamiltonian functional, $\delta \mathscr{H} / \delta \boldsymbol{u}$ is a column vector where each element is the functional derivative of the Hamiltonian functional by each element each element of $\boldsymbol{u}$ and $\boldsymbol{J}$ is a bilinear skew-symmetric mapping $(\boldsymbol{u}, \boldsymbol{J} \boldsymbol{v})=-(\boldsymbol{J} \boldsymbol{u}, \boldsymbol{v})$ and also satisfy the Jacobi identity $(2.13)$, where $(\cdot, \cdot)$ is the inner product of the space $\{\boldsymbol{u}\}$. Now let's define the function derivative (Greiner and Reinhardt, 1996).

Definition Let $M=\{\phi(x): x \in \mathbb{C}\}$ be a normed vector space. A functional $\mathscr{F}[\phi]$ is a mapping $\mathscr{F}: M \rightarrow \mathbb{C}$. The variation of a functional $\mathscr{F}[\phi]$ is defined as

$$
\begin{aligned}
\delta F[\phi] & =F[\phi+\delta \phi]-F[\phi] \\
& :=\int \frac{\delta F[\phi]}{\delta \phi(x)} \delta \phi(x) d x
\end{aligned}
$$

Just like in the discrete case, we can define the Casimir functionals $\mathscr{C}$. Analogous to (2.15), the Casimir of an infinite dimensional noncanonical Hamiltonian system is defined as

$$
\boldsymbol{J} \frac{\delta \mathscr{C}}{\delta \boldsymbol{u}}=0
$$

For an energy-conserving system, with the Casimir functional, it is possible to define an integral of motion called pseudo-energy. The pseudo-energy is just an integral of motion, but it is also quadratic to the lowest order. So, the pseudo-energy is positive-definite to the lowest order. The latter property is useful to analyze the stability of the system. The pseudo-energy $\mathscr{A}$ is defined as follows

$$
\mathscr{A}(\boldsymbol{u})=\mathscr{H}(\boldsymbol{u})-\mathscr{H}(\boldsymbol{U})+\mathscr{C}(\boldsymbol{u})-\mathscr{C}(\boldsymbol{U})
$$

where $\boldsymbol{U}$ is a stationary state of the system. Since $\boldsymbol{U}$ is stationary (i.e. $\boldsymbol{u}_{t}(\boldsymbol{u}=\boldsymbol{U})=0$ ), then

$$
\left.\boldsymbol{J} \frac{\delta \mathscr{H}}{\delta \boldsymbol{u}}\right|_{\boldsymbol{u}=\boldsymbol{U}}=\left.\boldsymbol{u}_{t}\right|_{\boldsymbol{u}=\boldsymbol{U}}=\left.0 \Rightarrow \frac{\delta \mathscr{H}}{\delta \boldsymbol{u}}\right|_{\boldsymbol{u}=\boldsymbol{U}}=-\left.\frac{\delta \mathscr{C}}{\delta \boldsymbol{u}}\right|_{\boldsymbol{u}=\boldsymbol{U}},
$$

so, the variation of $\mathscr{A}$ is

$$
\begin{aligned}
\delta \mathscr{A} & =\left.\mathscr{A}\right|_{u=\boldsymbol{U}}+\left(\left.\frac{\delta \mathscr{A}}{\delta \boldsymbol{u}}\right|_{\boldsymbol{u}=\boldsymbol{U}}, \delta \boldsymbol{u}\right)+\mathcal{O}\left(\delta \boldsymbol{u}^{2}\right), \\
& =0+\left(\left.\frac{\delta \mathscr{H}}{\delta \boldsymbol{u}}\right|_{\boldsymbol{u}=\boldsymbol{U}}+\left.\frac{\delta \mathscr{C}}{\delta \boldsymbol{u}}\right|_{\boldsymbol{u}=\boldsymbol{U}}, \delta \boldsymbol{u}\right)+\mathcal{O}\left(\delta \boldsymbol{u}^{2}\right), \\
& =\mathcal{O}\left(\delta \boldsymbol{u}^{2}\right) .
\end{aligned}
$$


Thus, the pseudo-energy is indeed quadratic to the lowest order.

Now we will apply the Hamiltonian formalism to the well-known rotating shallow water model(Shepherd, 1990).

Example The governing equations of a shallow, homogeneous fluid in a rotating reference frame, with a constant vertical angular speed $f / 2$, are

$$
\begin{aligned}
\frac{\partial \boldsymbol{v}}{\partial t}+(\boldsymbol{v} \cdot \boldsymbol{\nabla}) \boldsymbol{v}+f \hat{\boldsymbol{z}} \times \boldsymbol{v} & =-g \boldsymbol{\nabla} h \\
\frac{\partial h}{\partial t}+\boldsymbol{\nabla} \cdot(h \boldsymbol{v}) & =0
\end{aligned}
$$

where $\boldsymbol{v}(x, y, t)=(u(x, y, t), v(x, y, t))$ is the horizontal velocity, $h(x, y, t)$ is the fluid depth, $g$ is the gravity local acceleration, $\hat{z}$ is the vertical unit vector and the operator $\boldsymbol{\nabla}=$ $(\partial / \partial x, \partial / \partial y)$. The Hamiltonian system is defined by a state vector is $\boldsymbol{u}=(\boldsymbol{v}, h)^{T}$ and the Hamiltonian functional

$$
\mathscr{H}(\boldsymbol{v}, h)=\frac{1}{2} \iint\left(h|\boldsymbol{v}|^{2}+g h^{2}\right) d x d y
$$

and the operator $\boldsymbol{J}$ is defined as

$$
\boldsymbol{J}=\left(\begin{array}{ccc}
0 & q & -\frac{\partial}{\partial x} \\
-q & 0 & -\frac{\partial}{\partial y} \\
-\frac{\partial}{\partial x} & -\frac{\partial}{\partial y} & 0
\end{array}\right)
$$

where $q$ is the potential vorticity

$$
q \equiv \frac{(f+\hat{z} \cdot \nabla \times v)}{h} \equiv \frac{\omega}{h}
$$

The Casimir functional $\mathscr{C}$ in an infinite-dimensional dynamical system satisfy the following equation (Shepherd, 1990)

$$
\boldsymbol{J} \frac{\delta \mathscr{C}}{\partial \boldsymbol{u}}=0
$$


The Casimir functional of the rotating shallow water model is

$$
\mathscr{C}=\iint h C(q) d x d y
$$

where $C(q)$ is a function of $q$. Notice that the mass conservation and the circulation theorem are just particular cases of the general Casimir functional. Letting $C(q)=1$ and $C(q)=q$ yields the mass conservation and circulation theorem, respectively. Let verify that $\mathscr{C}$ is the Casimir functional of the system. The variation of $\mathscr{C}$ is

$$
\delta \mathscr{C}=\iint\left(h C^{\prime}(q) \delta q+C(q) \delta h\right) d x d y
$$

Since $q=\omega / h$, we have

$$
\delta q=\frac{h \delta \omega-\omega \delta h}{h^{2}}
$$

with the two equations above, it is straightforward to show that the variation of $\mathscr{C}$ is

$$
\delta \mathscr{C}=\iint\left[\frac{\partial C^{\prime}(q)}{\partial x} \delta v-\frac{\partial C^{\prime}(q)}{\partial y} \delta h+\left(\frac{\omega}{h} C^{\prime}(q)+C(q)\right) \delta h\right] d x d y
$$

Thus,

$$
\begin{aligned}
& \frac{\delta \mathscr{C}}{\delta v}=\frac{\partial C^{\prime}(q)}{\partial x} \\
& \frac{\delta \mathscr{C}}{\delta u}=-\frac{\partial C^{\prime}(q)}{\partial y} \\
& \frac{\delta \mathscr{C}}{\delta h}=\frac{\omega}{h} C^{\prime}(q)+C(q) .
\end{aligned}
$$

Finally, we show that $\mathscr{C}$ satisfy equation (2.35) 


$$
\begin{aligned}
& \boldsymbol{J} \frac{\delta \mathscr{C}}{\delta \boldsymbol{u}}=\left(\begin{array}{ccc}
0 & q & -\frac{\partial}{\partial x} \\
-q & 0 & -\frac{\partial}{\partial y} \\
-\frac{\partial}{\partial x} & -\frac{\partial}{\partial y} & 0
\end{array}\right)\left(\begin{array}{c}
\frac{\delta \mathscr{C}}{\delta u} \\
\frac{\delta \mathscr{C}}{\delta v} \\
\frac{\delta \mathscr{C}}{\delta h}
\end{array}\right) \\
& =\left(\begin{array}{c}
q \frac{\delta \mathscr{C}}{\delta v}-\frac{\partial}{\partial x} \frac{\delta \mathscr{C}}{\delta h} \\
-q \frac{\delta \mathscr{C}}{\delta u}-\frac{\partial}{\partial y} \frac{\delta \mathscr{C}}{\delta h} \\
-\frac{\partial}{\partial x} \frac{\delta \mathscr{C}}{\delta u}-\frac{\partial}{\partial y} \frac{\delta \mathscr{C}}{\delta v}
\end{array}\right) \\
& =\left(\begin{array}{l}
0 \\
0 \\
0
\end{array}\right)
\end{aligned}
$$

\subsection{Chapter Summary}

The material in this chapter is entirely a review and no claims to originality are made. 
Chapter 3

\section{Governing Equations and Normal Modes}

The model adopted here is the so-called shallow global non-hydrostatic model (White et al., 2005). This model can be obtained from the full Euler equations in a uniformly rotating frame and spherical-oblate coordinate system by approximating the geopotential surfaces by spheres and adopting some approximations that result from classical scaleanalysis for large-scale motions in the atmosphere. These approximations include: (i) neglecting the apparent acceleration terms proportional to the vertical velocity in the horizontal momentum equations, including both those associated with the curvature of the coordinate system and the Coriolis term proportional to the cosine of latitude in the zonal momentum equation, and (ii) disregarding all the apparent acceleration terms in the vertical momentum equation. Furthermore, the approximation of a shallow atmosphere assumes that its thickness is very small compared to the Earth's radius, which is regarded as a constant. The shallow non-hydrostatic model has a noncanonical Hamiltonian strutucture. In order to employ that formalism we need:

- A function space $H=\{\boldsymbol{u}\}$ with an inner product $(\cdot, \cdot): H \times H \rightarrow \mathbb{R}$;

- A Hamiltonian functional $\mathscr{H}: H \rightarrow \mathbb{R}$;

- A bilinear, skew-symmetric mapping $\boldsymbol{J}:(u, \boldsymbol{J} v) \mapsto(-\boldsymbol{J} u, v)$ that also satisfy the Jacobi identity (2.13).

- A set of equations that describe the dynamical evolution of the system,

$$
\boldsymbol{u}_{t}=\boldsymbol{J} \frac{\delta \mathscr{H}}{\delta \boldsymbol{u}} .
$$

One of the main advantages of using the Hamiltonian formalism is that one can employ Noether's theorem to determine the system's integrals of motion systematically. For 
example, given that the Hamiltonian functional has a translational time symmetry, a translational space symmetry or a particle relabelling symmetry, then Noether's theorem ensures that the energy, momentum and potential vorticity are integrals of motion of the system, respectively.

The Hamiltonian structure of the nonhydrostatic primitive equations is defined as follows (Morrison, 1998; Shepherd, 1990):

$$
\boldsymbol{u}=(\vec{v}, \rho, \eta)^{T}
$$

where $\boldsymbol{u}$ is the state vector, $\vec{v}, \rho$ and $\eta$ are the velocity field, density and entropy, respectively. The Jacobian operator is (Shepherd, 1990):

$$
\boldsymbol{J}=\left(\begin{array}{ccccc}
0 & \frac{1}{\rho} \omega_{3} & -\frac{1}{\rho} \omega_{2} & -\partial_{x} & \frac{1}{\rho} \eta_{x} \\
-\frac{1}{\rho} \omega_{3} & 0 & \frac{1}{\rho} \omega_{1} & -\partial_{y} & \frac{1}{\rho} \eta_{y} \\
\frac{1}{\rho} \omega_{2} & -\frac{1}{\rho} \omega_{1} & 0 & -\partial_{z} & \frac{1}{\rho} \eta_{z} \\
\partial_{x} & \partial_{y} & \partial_{z} & 0 & 0 \\
-\frac{1}{\rho} \eta_{x} & -\frac{1}{\rho} \eta_{y} & -\frac{1}{\rho} \eta_{z} & 0 & 0
\end{array}\right)
$$

where $\boldsymbol{\omega}=f \hat{\boldsymbol{z}}+\boldsymbol{\nabla} \times \boldsymbol{v}$ is the potential vorticity. Finally, the Hamiltonian functional of the model is

$$
\mathscr{H}=\iiint\left\{\frac{1}{2} \rho|\vec{v}|^{2}+U(\rho, \eta)+\rho g z\right\} d x d y d z
$$

where $U=U(\rho, \eta)=\rho c_{v} T$ is the internal energy of an ideal gas and $\rho g z$ is the gravity potential. Let's calculate the variational derivatives of the Hamiltonian (3.4). First, notice that

$$
\delta U=c_{v} T \delta \rho+c_{v} \rho \delta T
$$

From classical thermodynamics (Euler equation),

$$
c_{v} \delta T=T \delta \eta+\frac{R T}{\rho} \delta \rho,
$$


thus,

$$
\begin{aligned}
\delta U & =c_{v} T \delta \rho+c_{v} \rho\left(\frac{T}{c_{v}} \delta \eta+\frac{R T}{c_{v} \rho} \delta \rho\right) \\
& =c_{p} T \delta \rho+\rho T \delta \eta \\
& =\left(U+\frac{p}{\rho}\right) \delta \rho+\rho T \delta \eta,
\end{aligned}
$$

where we used that the enthalpy is $H=c_{p} T \equiv U+p / \rho$. So, the variational derivatives that we need are

$$
\begin{aligned}
& \frac{\delta \mathscr{H}}{\delta \vec{v}}=\rho \vec{v} \\
& \frac{\delta \mathscr{H}}{\delta \rho}=\frac{1}{2}|\vec{v}|^{2}+g z+U+p / \rho, \\
& \frac{\delta \mathscr{H}}{\delta \eta}=\rho T .
\end{aligned}
$$

Now we will calculate the pseudo-energy of the model. The Casimir of the model is Kuroda $(1990 b, a)$ :

$$
\mathscr{C}=\iiint \rho C(\eta, q) d V
$$

and the variational derivatives of the Casimir are (Shepherd, 1990)

$$
\begin{aligned}
& \frac{\delta \mathscr{C}}{\delta \vec{v}}=\nabla \times\left(C_{q} \nabla \eta\right) \\
& \frac{\delta \mathscr{C}}{\delta \rho}=C-q C_{q} \\
& \frac{\delta \mathscr{C}}{\delta \eta}=\rho C_{\eta}-\nabla \cdot\left(C_{q} \omega\right)
\end{aligned}
$$

Now, let's consider a resting, hydrostatic basic state,

$$
\begin{aligned}
\vec{v} & =0, \\
\eta & =\eta_{0}(z), \\
\rho & =\rho_{0}(z), \\
\frac{d p_{0}}{d z} & =-\rho_{0} g .
\end{aligned}
$$

Substituting (3.13) into (3.12), one can show that the Casimir for this basic state is (Shepherd, 1993):

$$
C\left(\eta_{0}\right)=-g z-U\left(\eta_{0}, \rho_{0}\right)-\frac{p_{0}}{\rho_{0}}
$$


Andrews (1981) showed that the system's variables are constant along equipotential fields. Thus, it is convenient to define the basic state functions as functions of the isentropic surface $\eta_{0}$

$$
\begin{aligned}
\rho_{0}(\vec{x}) & =\tilde{\rho}\left(\eta_{0}\right) \\
p_{0}(\vec{x}) & =\tilde{p}\left(\eta_{0}\right) \\
T_{0}(\vec{x}) & =\tilde{T}\left(\eta_{0}\right) \\
z & =\tilde{z}\left(\eta_{0}\right)
\end{aligned}
$$

With these variables redefinition, the Casimir becomes

$$
C(\eta)=-g \tilde{z}(\eta)-U(\eta, \tilde{\rho}(\eta))-\frac{\tilde{p}(\eta)}{\tilde{\rho}(\eta)}
$$

Using (2.26) shows that the expression of the pseudo-energy is

$$
\begin{aligned}
\mathscr{A}(u) & =\mathscr{H}(u)-\mathscr{H}(U)+\mathscr{C}(u)-\mathscr{C}(U), \\
& =\iiint d V\left\{\frac{1}{2} \rho|\vec{v}|^{2}+\left(\rho-\rho_{0}\right) g z+\rho U(\eta, \rho)\right. \\
& \left.-\rho_{0} U\left(\eta_{0}, \rho_{0}\right)+\rho C(\eta)-\rho_{0} C\left(\rho_{0}\right)\right\} .
\end{aligned}
$$

Notice that the kinetic part of $\mathscr{A}$ is already quadratic to the lowest order. Let's show that the remaining terms are also quadratic to the lowest order.

Substituting (3.16) in the available potential energy expression (i.e. non-kinetic part of the pseudo-energy)(Shepherd, 1993):

$$
\begin{aligned}
A P E= & \iiint d V\left\{\left(\rho-\rho_{0}\right) g z+\rho U(\eta, \rho)-\rho_{0} U\left(\eta_{0}, \rho_{0}\right)+\rho C(\eta)-\rho_{0} C\left(\eta_{0}\right)\right\} \\
= & \iiint d V\left\{\left(\rho-\rho_{0}\right) g z+\rho U(\eta, \rho)-\rho_{0} U\left(\eta_{0}, \rho_{0}\right)\right. \\
& \left.-\rho g \tilde{z}(\eta)-\rho U(\eta, \tilde{\rho}(\eta))-\frac{\tilde{p}(\eta)}{\tilde{\rho}(\eta)}+\rho_{0} g \tilde{z}\left(\eta_{0}\right)+\rho_{0} U\left(\eta_{0}, \tilde{\rho}\left(\eta_{0}\right)\right)+\rho_{0} \frac{\tilde{p}\left(\eta_{0}\right)}{\tilde{\rho}\left(\eta_{0}\right)}\right\} \\
= & \iiint d V\left\{\rho g \tilde{z}\left(\eta_{0}\right)-\rho g \tilde{z}(\eta)+\rho U(\eta, \rho)-\rho U(\eta, \tilde{\rho}(\eta))-\rho \frac{\tilde{p}(\eta)}{\tilde{\rho}(\eta)}+p_{0}\right\}
\end{aligned}
$$

Let's consider the available potential energy per mass unity, i.e., $A P E \rightarrow A P E / \rho$ :

$$
A P E=\iiint d V\left\{g \tilde{z}\left(\eta_{0}\right)-g \tilde{z}(\eta)+U(\eta, \rho)-U(\eta, \tilde{\rho}(\eta))-\frac{\tilde{p}(\eta)}{\tilde{\rho}(\eta)}+\frac{p_{0}}{\rho}\right\}
$$

Let $H=H(\eta, p)$ be the enthalpy, thus

$$
d H=\left(\frac{\partial H}{\partial \eta}\right)_{p} d \eta+\left(\frac{\partial H}{\partial p}\right)_{\eta} d p=T d \eta-\rho^{-1} d p \Rightarrow T d \eta=d H+\rho^{-1} d p
$$


So,

$$
\tilde{T}^{\prime}\left(\eta_{0}\right)=\tilde{H}^{\prime}\left(\eta_{0}, \tilde{p}\left(\eta_{0}\right)\right)-\tilde{\rho}^{-1}\left(\eta_{0}\right) \tilde{p}^{\prime}\left(\eta_{0}\right)=\tilde{H}^{\prime}\left(\eta_{0}, \tilde{p}\left(\eta_{0}\right)\right)+g \tilde{z}\left(\eta_{0}\right) .
$$

Integrating the last equation,

$$
\int_{\eta_{0}}^{\eta} \tilde{T}\left(\eta^{\prime}\right) d \eta^{\prime}=H(\eta, \tilde{p}(\eta))-H\left(\eta_{0}, \tilde{p}\left(\eta_{0}\right)\right)+g \tilde{z}(\eta)-g \tilde{z}\left(\eta_{0}\right)
$$

From classical thermodynamics,

$$
U=H-p \rho^{-1}
$$

Plugging (3.21) and (3.22) in (3.18) results in

$$
\begin{aligned}
A P E & =\iiint_{V} d V\left(H(\eta, p)-H\left(\eta, p_{0}\right)-\frac{p-p_{0}}{\rho}+H\left(\eta, p_{0}\right)-H\left(\eta_{0}, p_{0}\right)-\int_{\eta_{0}}^{\eta} \tilde{T}\left(\eta^{\prime}\right) d \eta^{\prime}\right) \\
& =\Pi_{1}+\Pi_{2}
\end{aligned}
$$

where $\Pi_{1}$ and $\Pi_{2}$ are defined as

$$
\begin{aligned}
\Pi_{1} & =\iiint_{V} d V\left(H(\eta, p)-H\left(\eta, p_{0}\right)-\frac{p-p_{0}}{\rho}\right) \\
\Pi_{2} & =\iiint_{V} d V\left(H\left(\eta, p_{0}\right)-H\left(\eta_{0}, p_{0}\right)-\int_{\eta_{0}}^{\eta} \tilde{T}\left(\eta^{\prime}\right) d \eta^{\prime}\right)
\end{aligned}
$$

For an ideal gas, we have

$$
\begin{aligned}
\rho^{-1} & =\kappa c_{p} e^{\eta / c_{p}} p^{-(1-\kappa)} \\
\kappa & =R / c_{p} \\
\eta & =c_{p} \log \theta \\
\theta & =T \frac{p_{\text {ref }}}{p} \\
H & =c_{p} T=e^{\eta / c_{p}} p^{\kappa} c_{p}
\end{aligned}
$$

where $R$ is the universal gas constant, $c_{p}$ is the specific heat at constant pressure, $\theta$ is the potential temperature and we will take the reference pressure as $p_{\text {ref }}=1$. So, for an ideal gas the expressions for $\Pi_{1}$ e $\Pi_{2}$ are (Andrews, 1981)

$$
\begin{aligned}
\Pi_{1} & =\iiint_{V} d V c_{p} e^{\eta / c_{p}} p_{0}^{\kappa} f\left(p / p_{0}\right) \\
\Pi_{2} & =\iiint_{V} d V c_{p} T_{0} h\left(\theta / \theta_{0}\right) \\
f(x) & =(1-\kappa) x^{\kappa}+\kappa x^{-(1-\kappa)}-1 \\
h(x) & =x-1-\log x
\end{aligned}
$$


Andrews (1981) showed that $\Pi_{1}$ e $\Pi_{2}$ are positive-definite functions to the lowest order.

Let's expand the available potential energy per mass unity

$$
\begin{aligned}
& \Pi_{1}=\iiint_{V} d V\left(\frac{1}{2 \rho_{0} C_{s}^{2}} p^{\prime 2}-\frac{4+\kappa}{6 \rho_{0} C_{s}^{2} p_{0}} p^{\prime 3}+\frac{1}{2 \rho_{0} p_{0} C_{s}^{2}} p^{\prime 3}+\mathcal{O}\left(p^{\prime 4}\right)\right) \\
& \Pi_{2}=\iiint_{V} d V\left(\frac{g^{2} \rho_{0}}{2 N^{2}}\left(\frac{\theta^{\prime}}{\theta_{0}}\right)^{2}+\frac{g^{2}}{2 N^{2}} \rho^{\prime}\left(\frac{\theta^{\prime}}{\theta_{0}}\right)^{2}-\frac{g^{2} \rho_{0}}{3 N^{2}}\left(\frac{\theta^{\prime}}{\theta_{0}}\right)^{3}\right)
\end{aligned}
$$

where $p^{\prime}=p-p_{0}, \rho^{\prime}=\rho-\rho_{0}$ and $\theta^{\prime}=\theta-\theta_{0}$. Thus, the pseudo-energy of the system is

$$
\begin{aligned}
\mathscr{A}(u) & =\iiint_{V} d V\left(\frac{1}{2} \rho\left|\vec{v}^{\prime}\right|^{2}+\frac{1}{2 \rho_{0} C_{s}^{2}} p^{\prime 2}+\frac{g^{2} \rho_{0}}{2 N^{2}}\left(\frac{\theta^{\prime}}{\theta_{0}}\right)^{2}\right) \\
& +\iiint_{V} d V\left(-\frac{4+\kappa}{6 \rho_{0} C_{s}^{2} p_{0}} p^{\prime 3}+\frac{1}{2 \rho_{0} p_{0} C_{s}^{2}} p^{\prime 3}+\frac{g^{2}}{2 N^{2}} \rho^{\prime}\left(\frac{\theta^{\prime}}{\theta_{0}}\right)^{2}-\frac{g^{2} \rho_{0}}{3 N^{2}}\left(\frac{\theta^{\prime}}{\theta_{0}}\right)^{3}\right) \\
& +\mathcal{O}\left(\theta^{\prime 4}\right)+\mathcal{O}\left(p^{\prime 4}\right)
\end{aligned}
$$

Consider the following variable transformation

$$
\theta^{\prime} \rightarrow \frac{\theta^{\prime}}{\theta_{0}} g \rho_{0}
$$

Now the pseudo-energy (3.28) becomes

$$
\begin{aligned}
\mathscr{A}(u) & =\iiint_{V} d V\left(\frac{1}{2} \rho_{0}\left|\vec{v}^{\prime}\right|^{2}+\frac{1}{2 \rho_{0} C_{s}^{2}} p^{\prime 2}+\frac{1}{2 \rho_{0} N^{2}} \theta^{\prime 2}\right) \\
& +\iiint_{V} d V\left(\frac{1}{2} \rho^{\prime}\left|\vec{v}^{\prime}\right|^{2}-\frac{4+\kappa}{6 \rho_{0} C_{s}^{2} p_{0}} p^{\prime 3}+\frac{1}{2 \rho_{0} p_{0} C_{s}^{2}} p^{\prime 3}+\frac{1}{2 \rho_{0}^{2} N^{2}} \rho^{\prime} \theta^{\prime 2}-\frac{1}{3 N^{2} \rho_{0}^{2} g} \theta^{\prime 3}\right) \\
& +\mathcal{O}\left(\theta^{\prime 4}\right)+\mathcal{O}\left(p^{\prime 4}\right) .
\end{aligned}
$$

So, the pseudoenergy can be written as

$$
\mathscr{A}=\mathscr{A}^{(2)}+\mathscr{A}^{(3)}+\mathcal{O}\left(u^{\prime}, v^{\prime}, w^{\prime}, p^{\prime}, \theta^{\prime}\right)^{4}=\mathrm{const}
$$

where its components are

$$
\begin{aligned}
& \mathscr{A}^{(2)}=\iiint_{V} d V\left(\frac{1}{2} \rho_{0}\left|\vec{v}^{\prime}\right|^{2}+\frac{1}{2 \rho_{0} C_{s}^{2}} p^{\prime 2}+\frac{1}{2 \rho_{0} N^{2}} \theta^{\prime 2}\right) \\
& \mathscr{A}^{(3)}=\iiint_{V} d V\left(\frac{1}{2} \rho^{\prime}\left|\vec{v}^{\prime}\right|^{2}-\frac{4+\kappa}{6 \rho_{0} C_{s}^{2} p_{0}} p^{\prime 3}+\frac{1}{2 \rho_{0} p_{0} C_{s}^{2}} p^{\prime 3}+\frac{1}{2 \rho_{0}^{2} N^{2}} \rho^{\prime} \theta^{\prime 2}-\frac{1}{3 N^{2} \rho_{0}^{2} g} \theta^{\prime 3}\right)
\end{aligned}
$$

The quadratic terms of the pseudo-energy (3.32) is exactly the norm proposed by Kasahara and Qian (2000). Kasahara and Qian (2000) obtained the quadratic terms of the pseudoenergy by direct manipulation of perturbation equations. 


\subsection{Governing Equations - Linear Problem}

The nonhydrostatic primitive equations can be obtained by substituting (3.2), (3.3) and (3.4) in (2.22)

$$
\begin{aligned}
\frac{d u}{d t}-f v & =-\frac{1}{\rho} \frac{\partial p}{\partial x} \\
\frac{d v}{d t}+f u & =-\frac{1}{\rho} \frac{\partial p}{\partial y} \\
\delta_{H} \frac{d w}{d t} & =-\frac{1}{\rho} \frac{\partial p}{\partial z}-g \\
\frac{d \rho}{d t}+\rho\left(\vec{\nabla} \cdot \vec{V}+\frac{\partial w}{\partial z}\right) & =0 \\
p & =\rho R T \\
\frac{d p}{d t} & =\gamma R T \frac{d \rho}{d t}
\end{aligned}
$$

where $x, y$ and $z$ are the zonal, meridional and vertical coordinates, recpectvely; $\delta_{H}$ is equal to 1 if the model is nonhydrostatic and 0 if it is hydrostaic, $T$ is temperature, $\rho$ is density, $p$ is pressure, $R$ is the universal gas constant for dry air, $\gamma=C_{p} / C_{v}$, where $C_{p}$ is the specific heat at constant pressure and $C_{v}$ is the specific heat at constant volume. The variable's domain is $\left\{\left[0, L_{x}\right] \times\left[0, L_{y}\right] \times\left[0, z_{T}\right] \times[0,+\infty]\right\}$. The differential operators are defined as

$$
\begin{aligned}
\frac{d}{d t} & =\frac{\partial}{\partial t}+\vec{V} \cdot \vec{\nabla}+w \frac{\partial}{\partial z}, \\
\vec{V} \cdot \vec{\nabla} & =u \frac{\partial}{\partial x}+v \frac{\partial}{\partial y}, \\
\vec{\nabla} \cdot \vec{V} & =\frac{\partial u}{\partial x}+\frac{\partial v}{\partial y} .
\end{aligned}
$$

$\vec{V}=(u, v)$ is the horizontal wind vector field, $w$ is the vertical wind component and $f=f_{0}$ is the Coriolis parameter in a mid-latitude $f$-plane approximation, where $f_{0}=2 \Omega \sin \phi_{0}$, $a$ is the Earth's and $\phi_{0}=\pi / 4$.

In order to obtain the normal modes of the model, we will express the dynamical 
variables of the model as perturbations around a basic state:

$$
\begin{aligned}
u(x, y, z, t) & =u_{0}(x, y, z, t)+u^{\prime}(x, y, z, t), \\
v(x, y, z, t) & =v_{0}(x, y, z, t)+v^{\prime}(x, y, z, t), \\
w(x, y, z, t) & =w_{0}(x, y, z, t)+w^{\prime}(x, y, z, t), \\
p(x, y, z, t) & =p_{0}(x, y, z, t)+p^{\prime}(x, y, z, t), \\
\rho(x, y, z, t) & =\rho_{0}(x, y, z, t)+\rho^{\prime}(x, y, z, t), \\
T(x, y, z, t) & =T_{0}(x, y, z, t)+T^{\prime}(x, y, z, t), \\
\theta^{\prime}(x, y, z, t) & =\frac{g}{C_{s}^{2}} p^{\prime}(x, y, z, t)-g \rho^{\prime}(x, y, z, t) .
\end{aligned}
$$

Here we choose a resting, isothermal, hydrostatic basic state

$$
\begin{aligned}
u_{0} & =v_{0}=w_{0}=0 \quad p_{0}=p_{0}(z), \quad \rho_{0}=\rho_{0}(z) \\
\frac{d p_{0}}{d z} & =-\rho_{0} g, \quad T=T_{0} .
\end{aligned}
$$

Just like Qian and Kasahara (2003), we will substitute (3.38) and (3.39) in (3.34), but retain the quadratic terms

$$
\begin{gathered}
\frac{\partial u^{\prime}}{\partial t}-f v^{\prime}+\frac{1}{\rho_{0}} \frac{\partial p^{\prime}}{\partial x}=-\vec{V}^{\prime} \cdot \nabla u^{\prime}-w^{\prime} \frac{\partial u^{\prime}}{\partial z}+\frac{\rho^{\prime}}{\rho_{0}^{2}} \frac{\partial p^{\prime}}{\partial x} \\
\frac{\partial v^{\prime}}{\partial t}+f u^{\prime}+\frac{1}{\rho_{0}} \frac{\partial p^{\prime}}{\partial y}=-\vec{V}^{\prime} \cdot \nabla v^{\prime}-w^{\prime} \frac{\partial v^{\prime}}{\partial z}+\frac{\rho^{\prime}}{\rho_{0}^{2}} \frac{\partial p^{\prime}}{\partial y} \\
\frac{\partial w^{\prime}}{\partial t}+\frac{1}{\rho_{0}} \frac{\partial p^{\prime}}{\partial z}+\frac{g}{C_{s}^{2} \rho_{0}} p^{\prime}-\frac{\theta^{\prime}}{\rho_{0}}=-\vec{V}^{\prime} \cdot \nabla w^{\prime}-w^{\prime} \frac{\partial w^{\prime}}{\partial z}+\frac{\rho^{\prime}}{\rho_{0}^{2}} \frac{\partial p^{\prime}}{\partial z}+g \frac{\rho^{\prime 2}}{\rho_{0}^{2}} \\
\frac{1}{C_{s}^{2}} \frac{\partial p^{\prime}}{\partial t}-\frac{g \rho_{0}}{C_{s}^{2}} w^{\prime}+\rho_{0}\left(\nabla \cdot \vec{V}^{\prime}+\frac{\partial w^{\prime}}{\partial z}\right)=-\frac{1}{C_{s}^{2}}\left(\vec{V}^{\prime} \cdot \nabla p^{\prime}+w^{\prime} \frac{\partial p^{\prime}}{\partial z}\right)+ \\
+\frac{1}{C_{s}^{2}} \frac{T^{\prime}}{T_{0}}\left(-g \rho_{0} w^{\prime}+\frac{\partial p^{\prime}}{\partial t}\right)-\rho^{\prime}\left(\nabla \cdot \vec{V}^{\prime}+\frac{\partial w^{\prime}}{\partial z}\right) \\
\frac{\partial \theta^{\prime}}{\partial t}+N^{2} w^{\prime} \rho_{0}=-\vec{V}^{\prime} \cdot \nabla \theta^{\prime}-w^{\prime} \frac{\partial \theta^{\prime}}{\partial z}+\frac{g}{C_{s}^{2}} \frac{T^{\prime}}{T_{0}}\left(-g \rho_{0} w^{\prime}+\frac{\partial p^{\prime}}{\partial t}\right)
\end{gathered}
$$

where $N$ is the Brunt-Vaisala frequency, $C_{s}$ is the speed of sound

$$
\begin{aligned}
N^{2} & =-g\left(\frac{1}{\rho_{0}} \frac{d \rho_{0}}{d z}+\frac{g}{C_{s}^{2}}\right)=\frac{g \kappa}{H} \\
C_{s}^{2} & =\gamma R T_{0} \\
\vec{V}^{\prime} \cdot \vec{\nabla} & =u^{\prime} \frac{\partial}{\partial x}+v^{\prime} \frac{\partial}{\partial y} \\
\vec{\nabla} \cdot \vec{V}^{\prime} & =\frac{\partial u^{\prime}}{\partial x}+\frac{\partial v^{\prime}}{\partial y}
\end{aligned}
$$


where $H=R T_{0} / g$ is the height scale for an isothermal atmosphere and $\kappa=R / C_{p}$. Equations (3.40) can be written in a more compact form using differential operators

$$
\frac{\partial \vec{u}}{\partial t}+\mathscr{L}(\vec{u})=\mathscr{N}(\vec{u}, \vec{u})
$$

where $\vec{u}=\left[u^{\prime}, v^{\prime}, w^{\prime}, p^{\prime}, \theta^{\prime}\right]^{T}$ is the state vector of the model, $\mathscr{L}$ is the linear differential operator

$$
\mathcal{L}=\left[\begin{array}{ccccc}
0 & -f & 0 & \rho_{0}^{-1} \partial_{x} & 0 \\
f & 0 & 0 & \rho_{0}^{-1} \partial_{y} & 0 \\
0 & 0 & 0 & \rho_{0}^{-1}\left(\partial z+g / C_{s}^{2}\right) & -\rho_{0}^{-} 1 \\
\rho_{0} C_{s}^{2} \partial_{x} & \rho_{0} C_{s}^{2} \partial_{y} & \rho_{0} C_{s}^{2}\left(\partial_{z}-g / C_{s}^{2}\right) & 0 & 0 \\
0 & 0 & \rho_{0} N^{2} & 0 & 0
\end{array}\right]
$$

and $\mathscr{N}(\vec{u}, \vec{u})$ is a vector with the nonlinear quadratic terms

$$
\mathcal{B}(\boldsymbol{\Psi}, \boldsymbol{\Psi})=\left[\begin{array}{c}
-\boldsymbol{V}^{\prime} \cdot \nabla u^{\prime}+\rho^{\prime} \partial_{x} p^{\prime} / \rho_{0}^{2} \\
-\boldsymbol{V}^{\prime} \cdot \nabla v^{\prime}+\rho^{\prime} \partial_{y} p^{\prime} / \rho_{0}^{2} \\
-\boldsymbol{V}^{\prime} \cdot \nabla w^{\prime}+\rho^{\prime} \partial_{z} p^{\prime} / \rho_{0}^{2}+g \rho^{\prime} / \rho_{0}^{2} \\
-\boldsymbol{V}^{\prime} \cdot \nabla p^{\prime}+p^{\prime}\left(\partial_{t} p^{\prime}-g \rho_{0} w^{\prime}\right) / p_{0} \\
-\boldsymbol{V}^{\prime} \cdot \nabla \theta^{\prime}+\left(g / C_{s}^{2}\right) p^{\prime}\left(\partial_{t} p^{\prime}-g \rho_{0} w^{\prime}\right) / p_{0}-g \rho^{\prime}\left(\partial_{t} \rho^{\prime} / \rho_{0}-w^{\prime} / H\right)
\end{array}\right]
$$

The normal modes $\vec{u}_{a}(x, y, z)$ are the characteristic solutions of the linearized equations, i.e., solutions of $(3.45)$ when $\mathscr{N}(\vec{u}, \vec{u})=0$. These modes are labeled with an index $a=$ $(m, n, k, r)$, where $m$ is the zonal wavenumber, $n$ is the meridional wavenumber, $k$ is the vertical wavenumber, and $r$ is the kind of oscillation. Qian and Kasahara (2003) described the normal modes of the nonhydrostatic primitive equations on a mid-latitude and equatorial beta planes.

\subsection{Separation of Variables}

To obtain the normal modes, we must solve the linearized version of equation (3.45)

$$
\frac{\partial \vec{u}}{\partial t}+\mathscr{L}(\vec{u})=0
$$


Following Qian and Kasahara (2003), a convenient separation of variables is

$$
\left[\begin{array}{c}
\rho_{0}^{1 / 2} u^{\prime} \\
\rho_{0}^{1 / 2} v^{\prime} \\
\rho_{0}^{-1 / 2} p^{\prime} \\
\rho_{0}^{1 / 2} w^{\prime} \\
\rho_{0}^{-1 / 2} \theta^{\prime} \\
\rho_{0}^{-1 / 2} \rho^{\prime} \\
\rho_{0}^{-1 / 2} T^{\prime}
\end{array}\right]=\left[\begin{array}{c}
U(y) \xi(z) \\
i V(y) \xi(z) \\
W(y) \xi(z) \\
i W(y) \eta(z) \\
W(y) \theta(z) \\
W(y) \zeta(z) \\
W(y) \chi(z)
\end{array}\right] \exp [i(m x-\omega t)]
$$

where $i=\sqrt{-1}$ is the imaginary unit, $m$ is the zonal wavenumber and $\omega$ is the natural time frequency. Plugging (3.49) in (3.48)

$$
\begin{aligned}
-\omega U-f V+m W & =0, \\
f U+\omega V+\frac{d W}{d y} & =0, \\
\frac{d \xi}{d z}+\frac{1}{2 \rho_{0}} \frac{d \rho_{0}}{d z} \xi+\delta_{H} \omega \eta+g \zeta & =0 \\
-\omega \theta+N^{2} \eta & =0, \\
-\frac{1}{C_{s}^{2}} W \xi+\left(m U+\frac{d V}{d y}\right) \xi+W L_{1}(\eta) & =0
\end{aligned}
$$

where

$$
\begin{aligned}
L_{1} & =\frac{d}{d z}-\Gamma \\
\Gamma & =\frac{1}{2 \rho_{0}} \frac{d \rho_{0}}{d z}+\frac{g}{C_{s}^{2}}=\frac{1}{2}\left(\frac{g}{C_{s}^{2}}-\frac{N^{2}}{g}\right) .
\end{aligned}
$$

and applying the following separability condition Kasahara and Qian (2000); Qian and Kasahara (2003),

$$
m U+\frac{d V}{d y}=\frac{\omega}{g h_{e}} W
$$

where $h_{e}$ is the separation parameter, also known as equivalent height. The equivalent height in a compressible and hydrostatic atmosphere is analogous to the depth of a homogeneous ocean Taylor (1936). We will also consider a rigid-lid boundary condition on the vertical direction

$$
\begin{gathered}
w^{\prime}(x, y, z=0, t)=w^{\prime}\left(x, y, z=z_{T}, t\right)=0 \\
\Rightarrow \eta(0)=\eta\left(z_{T}\right)=0
\end{gathered}
$$


After some manipulations, Qian and Kasahara (2003) showed that (3.50) and 3.53 reduces to the following vertical eigenvalue problem

$$
\begin{aligned}
L_{2}\left[\left(\frac{1}{C_{s}^{2}}-\frac{1}{g h_{e}}\right)^{-1} L_{1}(\eta)\right] & =\left(N^{2}-\delta_{H} \omega^{2}\right) \eta \\
\omega\left(-\frac{1}{C_{s}^{2}}+\frac{1}{g h_{e}}\right) \xi+L_{1}(\eta) & =0 \\
\theta & =\frac{N^{2}}{\omega} \eta \\
\zeta & =\frac{\xi}{C_{s}^{2}}-\frac{\theta}{g} \\
\chi & =\frac{\xi}{R T_{0}}-\zeta \\
L_{1} & =\frac{d}{d z}+\Gamma \\
L_{2} & =\frac{d}{d z}-\Gamma \\
\Gamma & =\frac{1}{2 \rho_{0}} \frac{d \rho_{0}}{d z}+\frac{g}{C_{s}^{2}} \\
\eta(0) & =\eta\left(z_{T}\right)=0
\end{aligned}
$$

Adding also a rigid-lid boundary condition in the meridional direction yields the following meridional eigenvalue problem (Qian and Kasahara, 2003)

$$
\begin{array}{r}
\frac{d^{2} V}{d y^{2}}+\left\{\frac{1}{g h_{e}}\left[\omega^{2}-\left(f_{0}+\beta y\right)^{2}\right]-\frac{m}{\omega} \beta-m^{2}\right\} V=0 \\
\left(\frac{d}{d y}-\frac{m f}{\omega}\right) V=\left(\frac{1}{g h_{e}}-\frac{m^{2}}{\omega^{2}}\right) \omega W \\
U=\frac{-\omega\left(f_{0}+\beta y\right) V+m g h_{e} d V / d y}{\omega^{2}-m^{2} g h_{e}} \\
V(0)=V\left(L_{y}\right)=0
\end{array}
$$

where (3.56a) is the Laplace's Tidal equation analogue in a cartesian geometry. The separation parameter $h_{e}$ is defined as follows

$$
h_{e}=\frac{C_{s}^{2}}{g}\left(1+\frac{C_{s}^{2} \lambda_{k_{i}}}{N^{2}-\delta_{H} \omega^{2}}\right)^{-1},
$$

and the eigenfrequencies $\omega$ can be obtained by solving

$$
\omega^{3}-\left[\left(m^{2}+n^{2}\right) g h_{e}+f_{0}^{2}\right] \omega-m \beta g h_{e}=0 .
$$




\subsection{Normal Modes on a $f$-plane.}

Qian and Kasahara (2003) showed that three kinds of normal modes characterize the nonhydrostatic shallow model on a $f$-plane. There are acoustic modes, inertia-gravity modes, Lamb modes. Acoustic modes have time frequency higher than the Brunt-Vaisala frequency. They are present only in nonhydrostatic, compressible models. Inertia-gravity modes' frequency is limited by the Brunt-Vaisala frequency. Long gravity waves are nearly hydrostatic, while short gravity waves exhibit nonhydrostatic effects. Lamb modes are present in both hydrostatic and nonhydrostatic models. They are characterized for having no vertical velocity, i.e., $w^{\prime}(z) \equiv 0$.

\subsubsection{Eigenfunctions Meridional and Vertical Structures}

Meridional eigenfunctions are easily obtained by solving the eigenvalue-eigenfunction problem (3.55). First, we solve (3.55a) with (3.55i) and obtain $\eta=\eta(z)$. Once $\eta(z)$ is known, the remaining eigenfunctions are easily obtained by direct substitution. The resulting eigenfunctions are

$$
\begin{aligned}
\eta_{i}(z) & =A_{k_{i}} \sin (k z) \\
\xi_{i}(z) & =\left(\frac{1}{C_{s}^{2}}-\frac{1}{g h_{e}}\right)^{-1} \frac{A_{k_{i}}}{\omega}[k \cos (k z)-\Gamma \sin (k z)] \\
\theta_{i}(z) & =\frac{N^{2}}{\omega} \eta_{i}(z) \\
\zeta_{i}(z) & =\frac{\xi_{i}(z)}{C_{s}^{2}}-\frac{\theta_{i}(z)}{g} \\
\chi_{i}(z) & =\frac{\xi_{i}(z)}{R T_{0}}-\zeta_{i}(z) \\
k & =\frac{k_{i} \pi}{z_{T}}, k_{i}=1,2,3 \ldots
\end{aligned}
$$


Analogously, solving (3.56) gives the following set of meridional eigenfunctions

$$
\begin{aligned}
V_{i}(y) & =A_{n_{i}} \sin (n y) \\
U_{i}(y) & =A_{n_{i}} \frac{-\omega f_{0} \sin (n y)+m n g h_{e} \cos (n y)}{\omega^{2}-m^{2} g h_{e}} \\
W_{i}(y) & =\frac{A_{n_{i}}}{\omega}\left(\frac{1}{g h_{e}}-\frac{m^{2}}{\omega^{2}}\right)^{-1}\left[n \cos (n y)-\frac{m}{\omega} f_{0} \sin (n y)\right] \\
n & =\frac{n_{i} \pi}{L_{y}}, n_{i}=1,2,3 \ldots \\
m & =\frac{2 m_{i} \pi}{L_{x}}, m_{i}=1,2,3 \ldots
\end{aligned}
$$

With the $f$-plane approximation, equations (3.57) and (3.58) can be solved analytically. The resulting eigenfrequencies are (Qian and Kasahara, 2003):

$$
\begin{aligned}
& \left.\begin{array}{l}
\omega_{a}^{2} \\
\omega_{g}^{2}
\end{array}\right\}=\frac{1}{2} C_{s}^{2}\left[m^{2}+n^{2}+k^{2}+\left(\frac{1}{2 H}\right)^{2}+f_{0}^{2} C_{s}^{-2}\right] \\
& \times\left\{1 \pm \sqrt{1-\frac{4\left[N^{2}\left(m^{2}+n^{2}\right)+\left(k^{2}+\left(\frac{1}{2 H}\right)\right) f_{0}^{2}\right]}{C_{s}^{2}\left[m^{2}+n^{2}+k^{2}+\left(\frac{1}{2 H}\right)^{2}+f_{0}^{2} C_{s}^{-2}\right]}}\right\}
\end{aligned}
$$

where $\omega_{a}$ and $\omega_{g}$ are the acoustic waves frequency and gravity wave frequency, respectively.

\subsubsection{Eigenfunctions Orthogonality and Completeness}

For the linearized equations the quadratic terms of the pseudo-energy (3.32) is exactly conserved. The conservation of (3.32) implies that the linear eigenmodes satisfy the following orthogonality relation (Kasahara and Qian 2000)

$$
i\left(\omega_{a}-\omega_{b}\right)\left\langle\vec{u}_{a}, \vec{u}_{b}\right\rangle_{\mathcal{A}^{(2)}}=0,
$$

where $\vec{u}_{a}$ and $\vec{u}_{b}$ represent two arbitrary eigenvectors whose components are defined by (3.48) and $\langle\cdot, \cdot\rangle_{\mathcal{A}^{(2)}}$ refers to the inner product in terms of pseudoenergy $\mathcal{A}^{(2)}$, given by

$$
\left\langle\vec{u}_{a}, \vec{u}_{b}\right\rangle_{\mathcal{A}^{(2)}}=\int_{0}^{z_{T}} \int_{0}^{L_{x}} \int_{0}^{L_{y}}\left[\rho_{0}\left(u_{a}^{*} u_{b}+v_{a}^{*} v_{b}+w_{a}^{*} w_{b}\right)+\frac{p_{a}^{*} p_{b}}{\rho_{0} C_{s}^{2}}+\frac{\theta_{a}^{*} \theta_{b}}{\rho_{0} N^{2}}\right] d y d x d z
$$

with the superscript "*"indicating the complex conjugate.

With the inner product (3.63), we can define the norm/energy $E_{a}$ of a mode as

$$
E_{a}:=\left\langle\vec{u}_{a}, \vec{u}_{a}\right\rangle_{\mathcal{A}^{(2)}}=K E U_{a}+K E V_{a}+K E W_{a}+T H E_{a}+A E E_{a}
$$


where the energy $E_{a}$ is partitioned into zonal kinetic energy, meridional kinetic energy, vertical kinetic energy, thermobaric energy and available elastic energy. Their respective expressions are

$$
\begin{aligned}
K E U_{a} & =\iiint_{V} \rho_{0}\left|u_{a}\right|^{2} d V=A_{k}^{2} A_{n}^{2} \frac{\left(\Gamma^{2}+k^{2}\right)\left(f_{0}^{2} \omega^{2}+g^{2} h_{e}^{2} m^{2} n^{2}\right)}{\omega^{2}\left(\frac{1}{C_{s}^{2}}-\frac{g}{h_{e}}\right)^{2}\left(\omega^{2}-g h_{e} m^{2}\right)^{2}} \\
K E V_{a} & =\iiint_{V} \rho_{0}\left|v_{a}\right|^{2} d V=A_{k}^{2} A_{n}^{2} \frac{C_{s}^{2} g^{2} h_{e}^{2}\left(\Gamma^{2}+k^{2}\right)}{\omega^{2}\left(C_{s}^{2}-g h_{e}\right)^{2}} \\
K E W_{a} & =\iiint_{V} \rho_{0}\left|w_{a}\right|^{2} d V=A_{k}^{2} A_{n}^{2} g^{2} h_{e}^{2}\left(\frac{1}{\omega^{2}-g h_{e} m^{2}}\right)^{2}\left(f_{0}^{2} m^{2}+n^{2} \omega^{2}\right), \\
T H E_{a} & =\iiint_{V} \frac{\left|p_{a}\right|^{2}}{\rho_{0} C_{s}^{2}} d V=A_{k}^{2} A_{n}^{2} g^{2} h_{e}^{2} N^{2} \frac{f_{0}^{2} m^{2}+n^{2} \omega^{2}}{N^{2}\left(\omega^{3}-g h_{e} m^{2} \omega\right)^{2}}, \\
A E E_{a} & =\iiint_{V} \frac{\left|\theta_{a}\right|^{2}}{\rho_{0} N^{2}} d V=A_{k}^{2} A_{n}^{2} g^{4} h_{e}^{4} C_{s}^{2} \frac{\left(\Gamma^{2}+k^{2}\right)\left(f_{0}^{2} m^{2}+n^{2} \omega^{2}\right)}{\omega^{2}\left(C_{s}^{2}-g h_{e}\right)^{2}\left(\omega^{2}-g h_{e} m^{2}\right)^{2}} .
\end{aligned}
$$

\subsection{Linear Dispersion}

The linear dispersion of the eigenmodes and their equivalent depths are shown in Figures 3.1 and 3.3, respectively.The dispersion curves (Fig. 3.1) are equations (3.61) and we plug these frequency values in (3.57) to obtain Figure 3.3.

There is a clear separation of scale between acoustic modes and gravity modes (Figs. 3.1 and 3.3). Acoustic modes' frequency is always higher than the Brunt-Vaisala frequency. Gravity modes' frequency is limited by the Brunt-Vaisala frequency. The equivalent depths of these modes are separated by the equivalent depth of the external mode $H_{\text {ext }}=7 \mathrm{H} / 5$ (Kasahara and Qian, 2000; Qian and Kasahara, 2003).

Figure 3.2 shows that long gravity modes have the same dispersion properties of hydrostatically balanced gravity waves. Nonhydrostatic effects are relevant for shorter gravity waves. For vertically internal modes remain hydrostatic even for shorter gravity modes.

\subsection{Normal Modes Energetics}

In this section we will analyze the energy partition (3.65) of acoustic and gravity modes.

\subsubsection{Gravity Modes}

Figure 3.4 shows that nonhydrostatic gravity waves have more vertical kinetic energy than horizontal kinetic energy. Vertically internal gravity haves retain their hydrostatic 


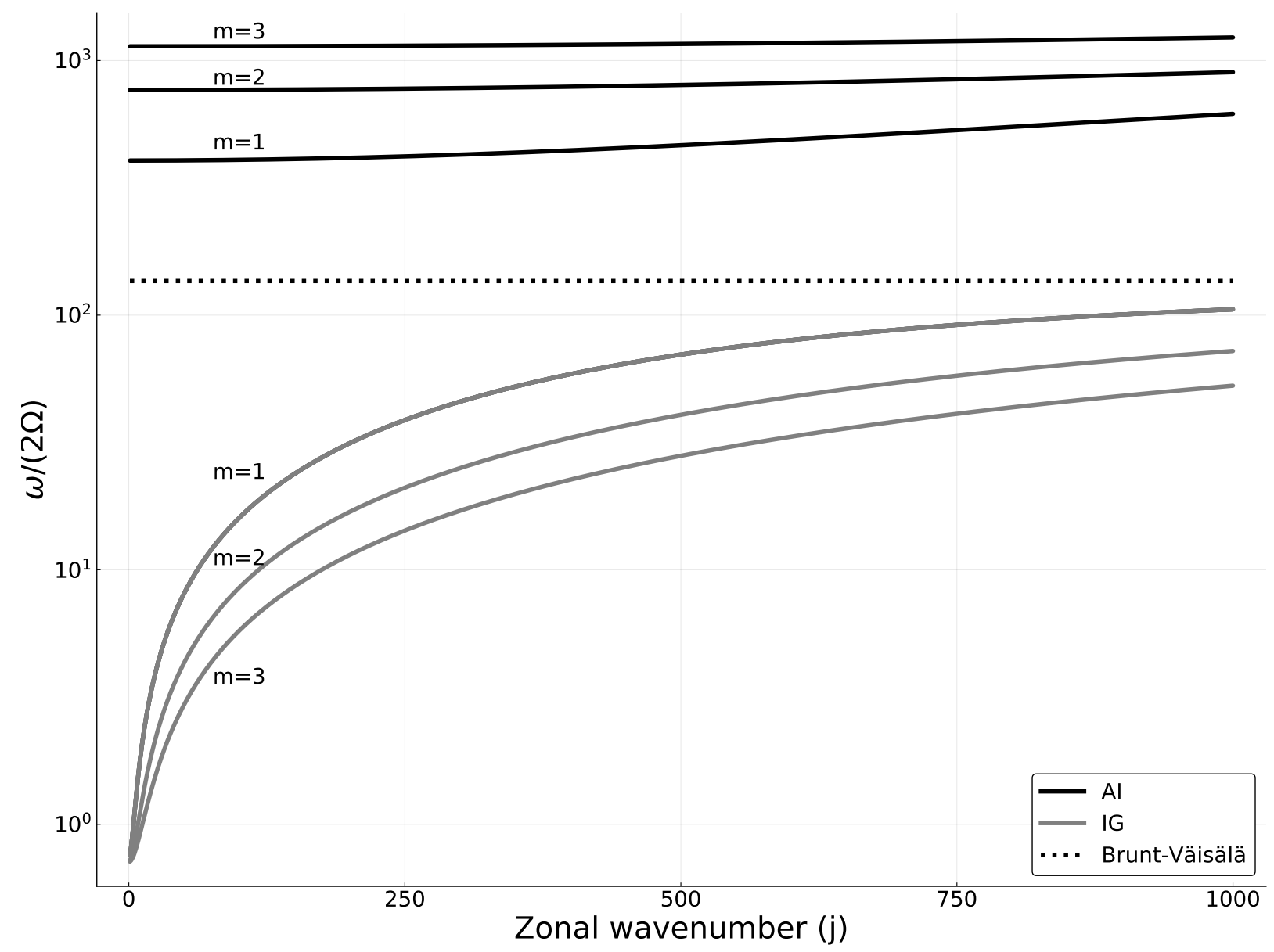

Figure 3.1: Dispersion curves of inertia-acoustic (IA) and inertia-gravity (IG) waves corresponding to the first three baroclinic modes $k=1,2,3$. All the curves are referred to the meridional index $n=1$. In this figure $j$ is the zonal wave index, $m$ is the vertical wave index.

characteristics even for small zonal scales (3.5). Long gravity waves with high meridional index (Fig. 3.6) have more meridional kinetic energy than long gravity waves with lower meridional index (Fig. 3.4). But, short (i.e., nonhydrostatic) gravity waves have the same energy partition, regardless of their meridional index.

\subsubsection{Acoustic Modes}

Long acoustic modes energy is dominated by available elastic energy (AEE) and vertical kinetic energy (KEW) (Fig. 3.7). Short acoustic waves still have the majority of its energy in the form of AEE, but their kinetic energy is concentrated in the form of zonal kinetic energy (KEU).

Long and vertically internal acoustic waves present equipartition of energy between AEE and KEW 3.8. This happens because the thermobaric (THE) portion of its energy is shifted towards its AEE. However, the inversion of roles between KEU and KEW occurs 


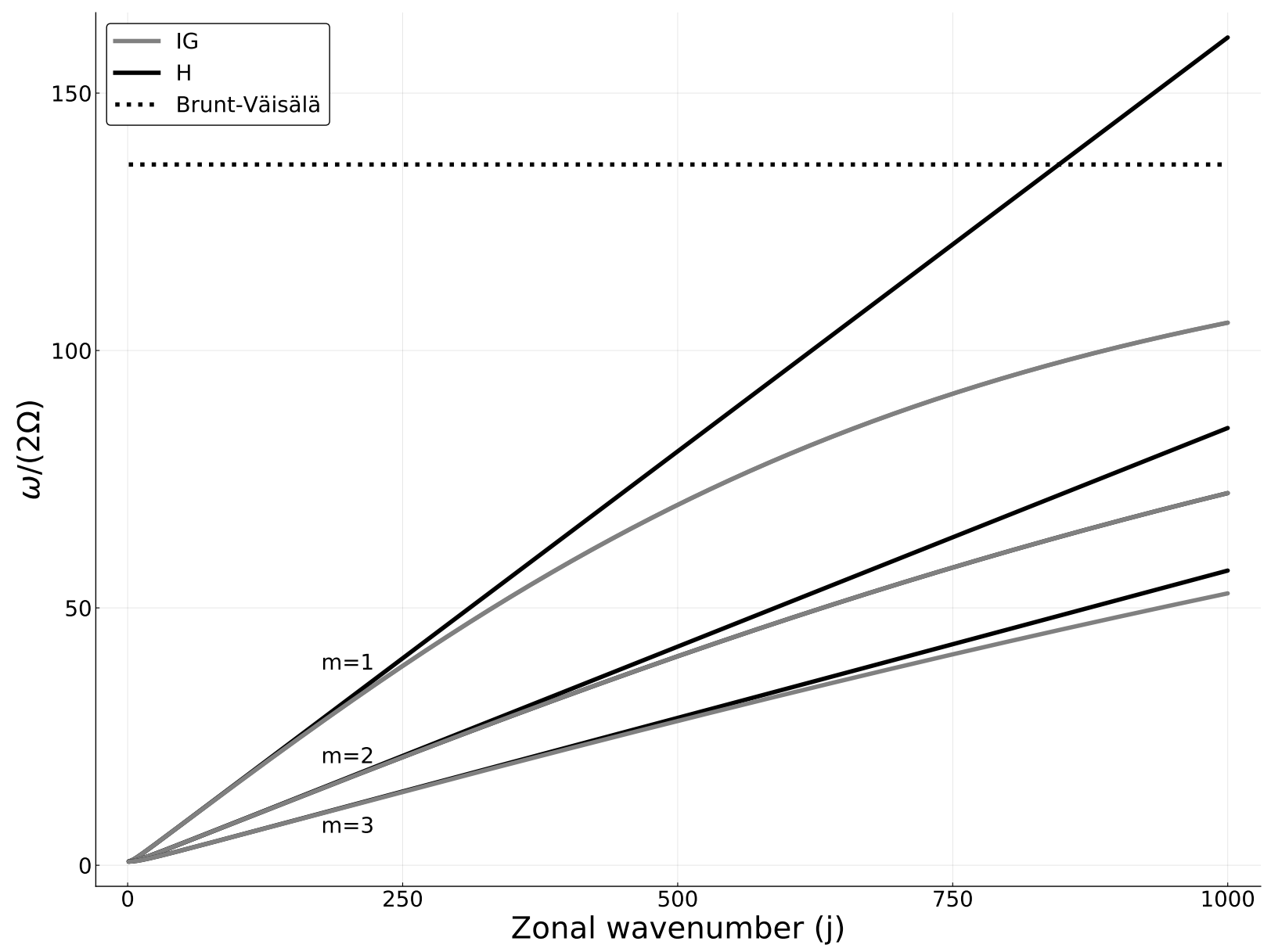

Figure 3.2: Similar to Fig. 3.1, but only for the inertia-gravity waves (IG) and their correspondent dispersion curves obtained by hydrostatic approximation $(\mathrm{H})$.

only for shorter acoustic modes.

Acoustic modes with low and high meridional index are very similar. However, for long acoustic modes, with high meridional index, the meridional kinetic energy (KEV) partition is more significant than the zonal kinetic energy (KEU). Short acoustic modes' energy partition is similar for low and high meridional index. 


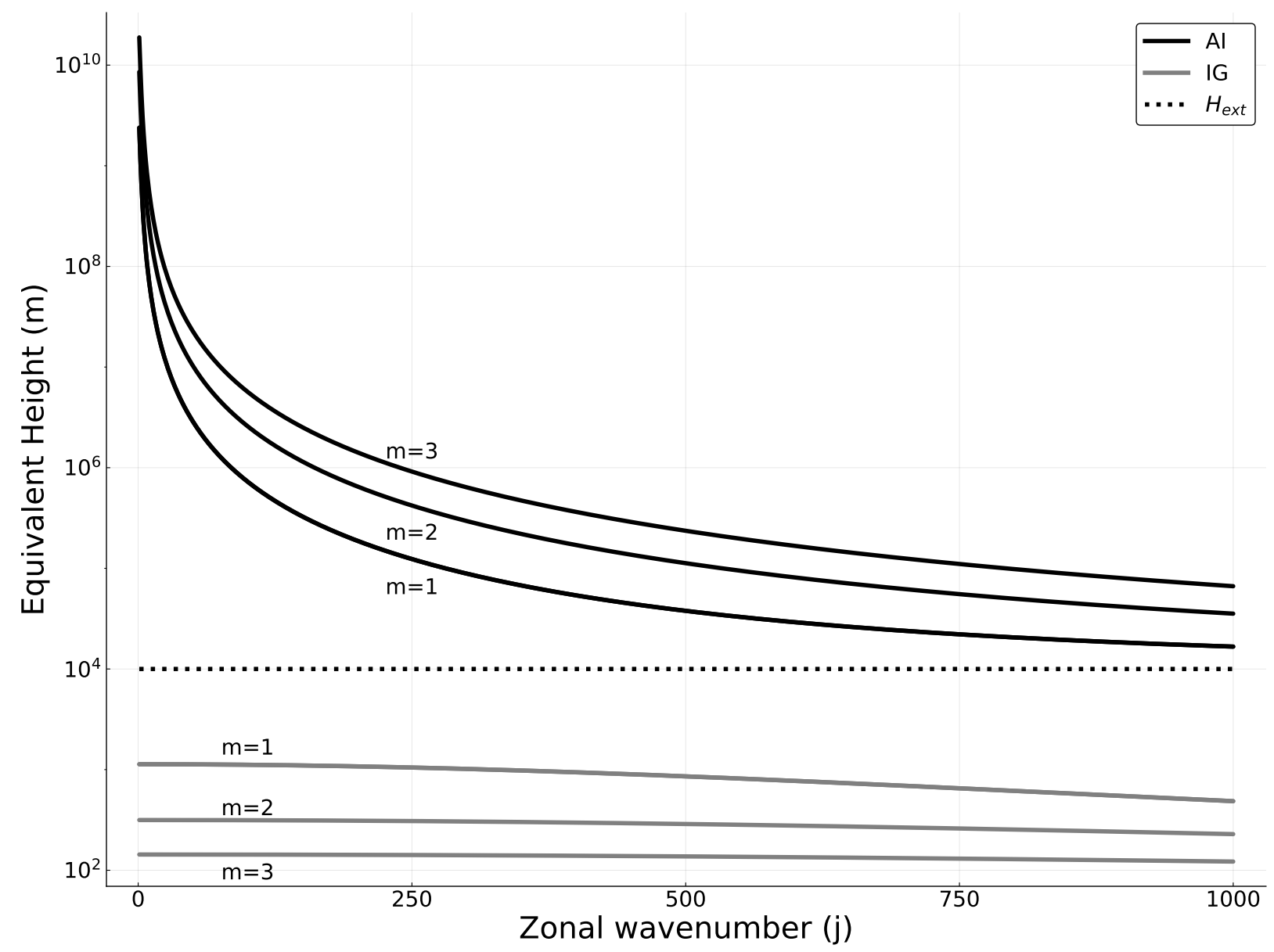

Figure 3.3: Equivalent height of acoustic (AI) and gravity modes (IG). In this figure $j$ is the zonal wave index, $m$ is the vertical wave index. All curves are for meridional index 1 . 


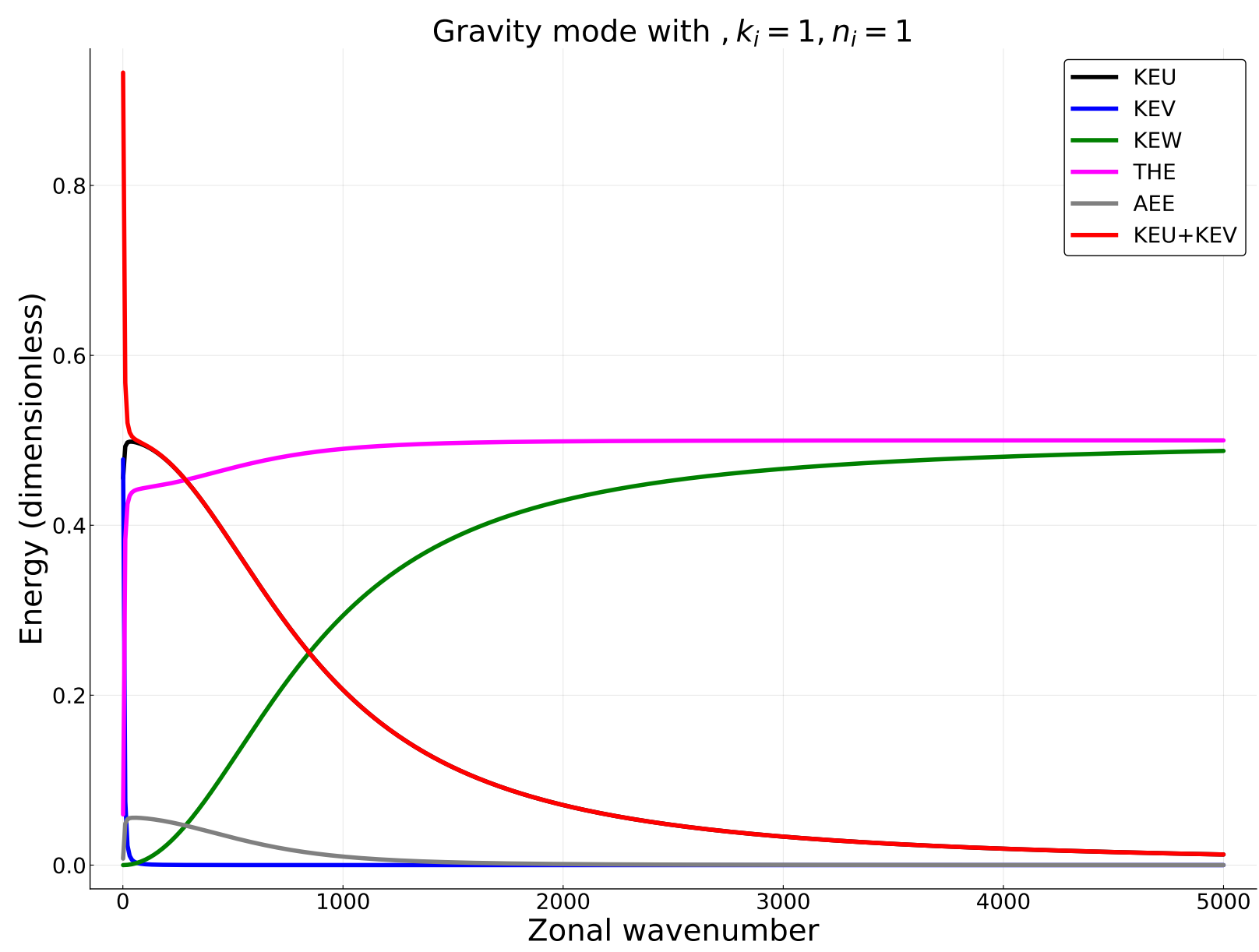

Figure 3.4: Gravity modes energy partition for vertical mode $k_{i}=1$ and meridional index $n_{i}=1$. 


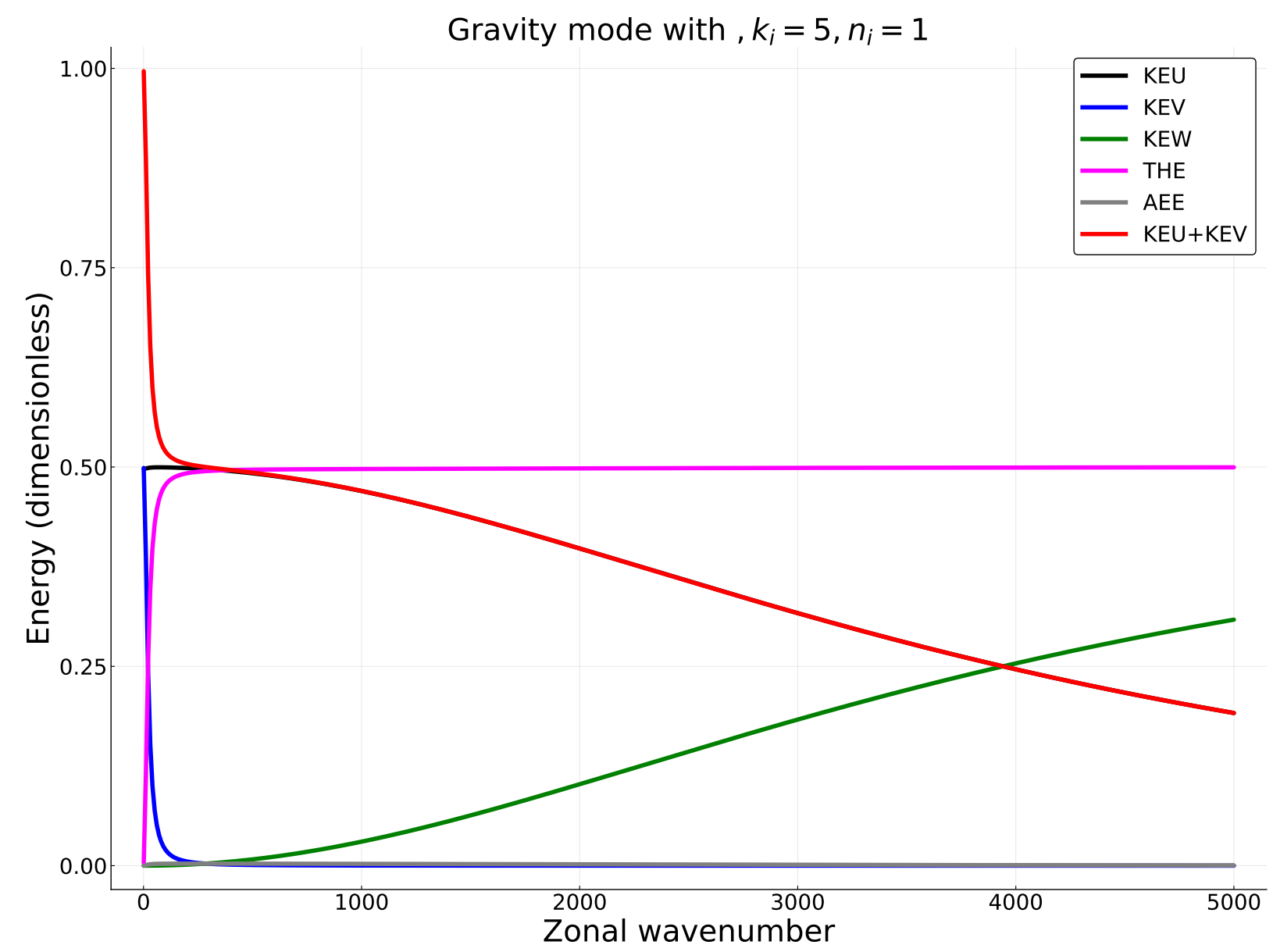

Figure 3.5: Gravity modes energy partition for vertical mode $k_{i}=5$ and meridional index $n_{i}=1$. 


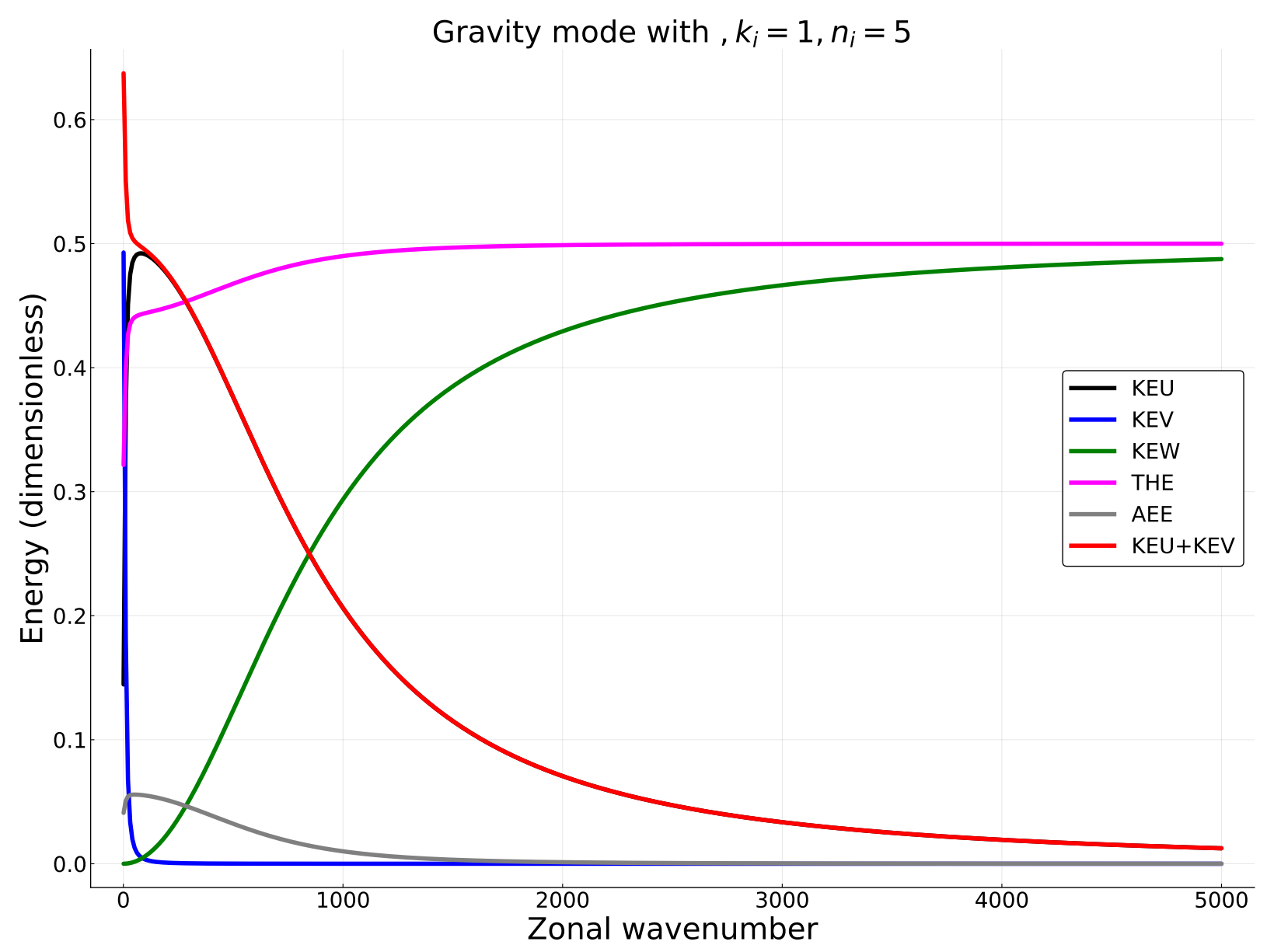

Figure 3.6: Gravity modes energy partition for vertical mode $k_{i}=1$ and meridional index $n_{i}=5$. 


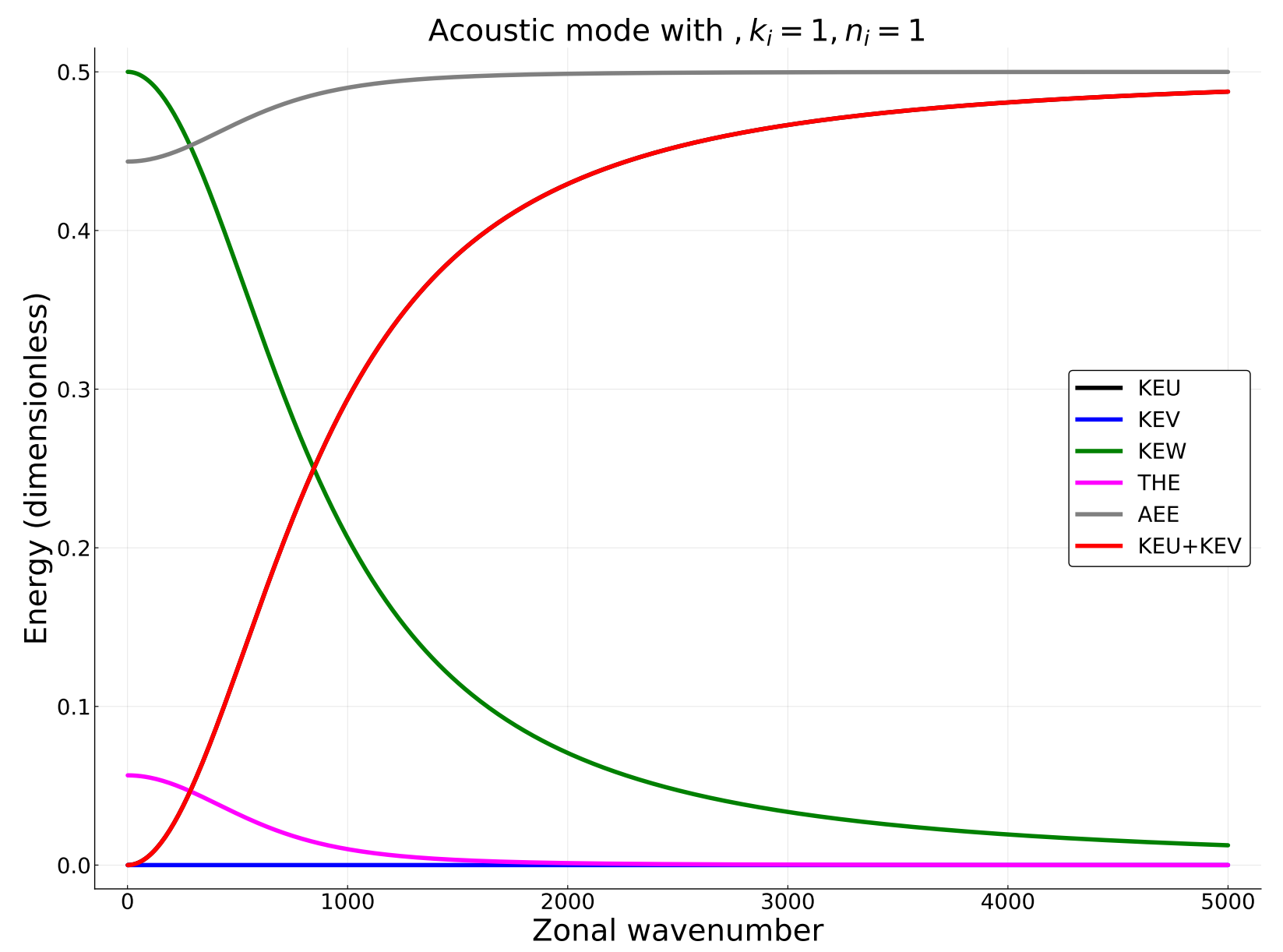

Figure 3.7: Acoustic modes energy partition for vertical mode $k_{i}=1$ and meridional index $n_{i}=1$. 


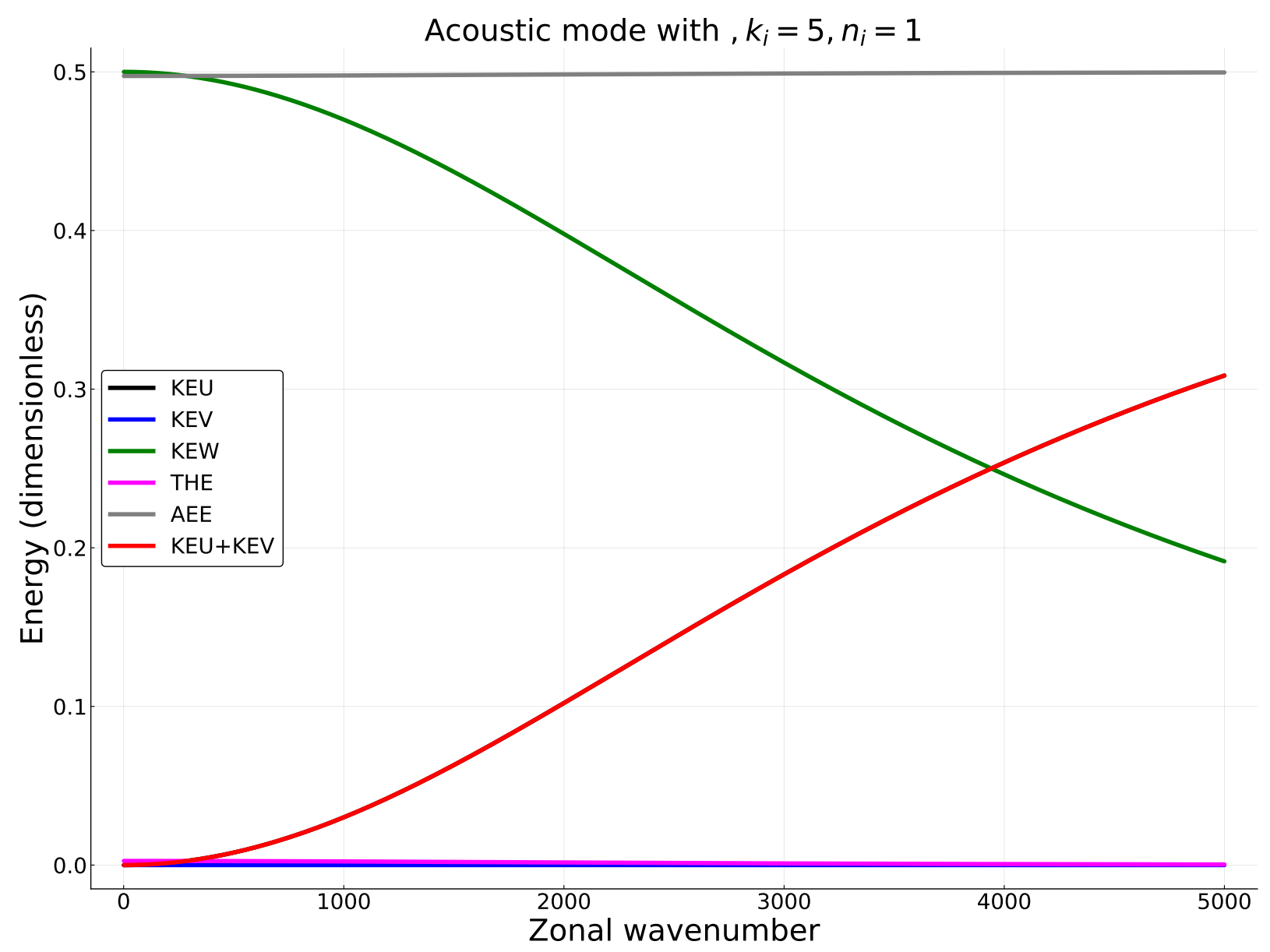

Figure 3.8: Acoustic modes energy partition for vertical mode $k_{i}=5$ and meridional index $n_{i}=1$. 


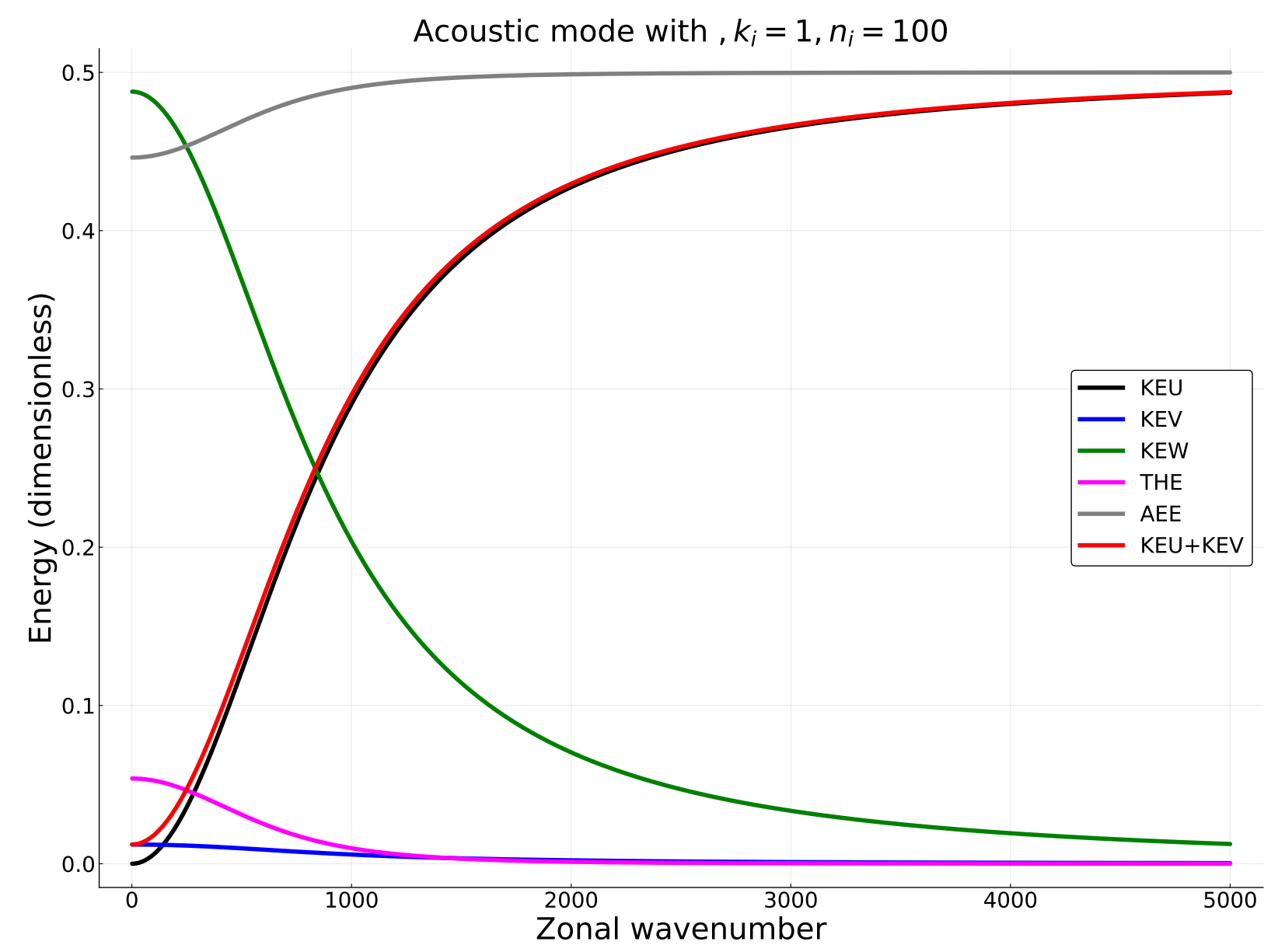

Figure 3.9: Acoustic modes energy partition for vertical mode $k_{i}=1$ and meridional index $n_{i}=100$. 
Chapter 4

\section{Weakly Nonlinear Dynamics}

\subsection{Modal expansion}

Now we use the orthogonality and completeness of the linear eigenmode functions described in the previous chapter to expand the solution of our nonlinear system (3.45) in a series

$$
\mathbf{u}=\left[\begin{array}{c}
u^{\prime}(x, y, z, t) \\
v^{\prime}(x, y, z, t) \\
w^{\prime}(x, y, z, t) \\
p^{\prime}(x, y, z, t) \\
\theta^{\prime}(x, y, z, t)
\end{array}\right]=\sum_{a} A_{a}(t) \mathbf{u}_{\mathbf{a}}+\text { C.C. }
$$

where "C.C." indicates the complex conjugate of what is preceding, $A_{a}(t)$ refers to the complex-valued spectral amplitudes and the vector $\mathbf{u}_{\mathbf{a}}=\left[u_{a}, v_{a}, w_{a}, p_{a}, \theta_{a}\right]^{T}$ represents the eigenvector function of a particular mode that is obtained by solving

$$
i \omega_{a} \boldsymbol{u}_{a}=\mathscr{L} \boldsymbol{u}_{a}
$$

with the boundary conditions discussed in the last chapter.

The expansion series (4.1) is an exact solution of system (3.45) provided the mode amplitudes $A_{a}(t)$ satisfy the following equation

$$
E_{a} A_{a}^{\prime}(t)=\sum_{b} \sum_{c} \sigma_{a}^{b c} A_{b} A_{c} e^{-i \delta_{a b c} t}
$$

where

$$
E_{a}=\left\langle\mathbf{u}_{\mathbf{a}}, \mathbf{u}_{\mathbf{a}}\right\rangle_{\mathcal{A}^{(2)}}
$$


is the intrinsic energy (i.e., the squared norm) of the $a$-th mode and $\delta_{a b c}=\omega_{a}-\omega_{b}-\omega_{c}$ represents the mismatch among the linear eigenfrequencies of each mode triplet $a b c$. If $\delta_{a b c}=0$, the triad is said to be resonant; the coupling constants $\sigma_{a}^{b c}$ are the projection of the nonlinear terms due to the action of two modes $b$ and $c$ onto another mode $a$, i.e.,

$$
\sigma_{c}^{a b}=\left\langle\mathbf{u}_{c}, \mathscr{N}\left(\mathbf{u}_{\mathbf{a}}, \mathbf{u}_{\mathbf{b}}\right)+\mathscr{N}\left(\mathbf{u}_{\mathbf{b}}, \mathbf{u}_{\mathbf{a}}\right)\right\rangle_{\mathcal{A}^{(2)}}
$$

where $\mathscr{N}$ is the bilinear operator containing the nonlinear terms of system (3.45), so all the information on the nonlinearity of our model equations is contained in these coefficients. Explicitly,

$$
\begin{aligned}
2 \sigma_{c}^{a b}: & =\iiint d x d y d z\left\{\left(-u_{a} \partial_{x} u_{b}-v_{a} \partial_{y} u_{b}-w_{a} \partial_{z} u_{b}+\frac{1}{\rho_{0}^{2}} \rho_{a} \partial_{x} p_{b}+(a \leftrightarrow b)\right) u_{c}^{*} \rho_{0}+\right. \\
& \left(-u_{a} \partial_{x} v_{b}-v_{a} \partial_{y} v_{b}-w_{a} \partial_{z} v_{b}+\frac{1}{\rho_{0}^{2}} \rho_{a} \partial_{y} p_{b}+(a \leftrightarrow b)\right) v_{c}^{*} \rho_{0}+ \\
& \left(-u_{a} \partial_{x} w_{b}-v_{a} \partial_{y} w_{b}-w_{a} \partial_{z} w_{b}+\frac{1}{\rho_{0}^{2}} \rho_{a} \partial_{z} p_{b}+g \frac{\rho_{a} \rho_{b}}{\rho_{0}^{2}}+(a \leftrightarrow b)\right) w_{c}^{*}+ \\
& \left(-u_{a} \partial_{x} p_{b}-v_{a} \partial_{y} p_{b}-w_{a} \partial_{z} p_{b}-\frac{p_{a}}{p_{0}}\left(\partial_{t} p_{b}-g \rho_{0} w_{b}\right)+(a \leftrightarrow b)\right) \frac{p_{c}^{*}}{\rho_{0} C_{s}^{2}}+ \\
& \left.\left(-u_{a} \partial_{x} \theta_{b}-v_{a} \partial_{y} \theta_{b}-w_{a} \partial_{z} \theta_{b}+\frac{g}{C_{s}^{2}} \frac{p_{a}}{p_{0}}\left(\partial_{t} p_{b}-g \rho_{0} w_{b}\right)-g \frac{\rho_{a}}{\rho_{0}}\left(\partial_{t} \rho_{b}+w_{b} \partial_{z} \rho_{0}\right)+(a \leftrightarrow b)\right) \frac{\theta_{c}^{*}}{\rho_{0} N^{2}}\right\} .
\end{aligned}
$$

where $(a \leftrightarrow b)$ means the same term as what is preceding but with the permutation of the indices $a$ and $b$. The interacting triads are those whose coupling constants are nonzero. The orthogonality of the $(x, y)$ basis functions (i.e. complex exponential in $x$ and trigonometric functions in $y$ ) requires the wave modes of an interacting triad to satisfy

$$
\begin{gathered}
m_{a}=m_{b}+m_{c}, \\
n_{a}=n_{b}+n_{c} .
\end{gathered}
$$

In addition, the vertical coupling integrals involved in (4.5) appear in the form

$$
\int_{0}^{z_{T}} \rho_{0}^{-\frac{1}{2}} \cos \left[\left(k_{a} \pm k_{b} \pm k_{c}\right) z\right] d z
$$

Thus, due to the presence of the "weight function" $\rho_{0}^{-\frac{1}{2}}$ in the vertical coupling constants, unlike the horizontal wavenumbers, there is no an excluding selection rule imposed by the vertical structures of the triad components. However, as $\rho_{0}$ is a monotonically decreasing 
function of $z$, if $k_{a} \pm k_{b} \pm k_{c} \neq 0$ the vertical coupling integrals will be small, so that the triads whose wave modes do satisfy the condition

$$
k_{a} \pm k_{b} \pm k_{c}=0
$$

together with conditions (4.7), undergo the strongest interactions, even though condition (4.8) is no longer excluding. These wave triplets shall be called hereafter as interacting triads.

\subsection{Energy constraints for the interacting triads}

As explained by Ripa (1981) in the context of barotropic Rossby waves and internal gravity waves in a vertical plane and Ripa (1983a) and Vanneste and Vial (1994) for the equatorial beta-plane and spherical geometry shallow-water equations, respectively, the conserved quantities which are quadratic to lowest order in terms of wave disturbances lead to relations among the coupling constants of an interacting triad. Thus, in what follows we shall apply their approach in our nonhydrostatic context. In this way, substituting the mode expansion (4.1) into (3.32) and (3.33) yields:

$$
\begin{aligned}
& \mathscr{A}^{(2)}=\sum_{a} E_{a}\left|A_{a}\right|^{2} \\
& \mathscr{A}^{(3)}=\sum_{a} \sum_{b} \sum_{c} 2 S_{a b c} \operatorname{Re}\left(A_{a} A_{b}^{*} A_{c}^{*} e^{i \delta_{a b c} t}\right)
\end{aligned}
$$

Equations above show that, as a consequence of the orthogonality relation (3.62), the leading-order (quadratic) pseudoenergy has a diagonalized representation in terms of the linear eigenmodes, whilst the cubic pseudoenergy $\mathscr{A}^{(3)}$ is expanded in terms of all interacting triads, with coefficients $S_{a b c}$ being given by

$$
\begin{aligned}
S_{a b c} & =\int_{0}^{z_{T}} \int_{0}^{L_{x}} \int_{0}^{L_{y}}\left\{\frac{1}{2} \rho_{a}\left(u_{b}^{*} u_{c}^{*}+v_{b}^{*} v_{c}^{*}+w_{b}^{*} w_{c}^{*}\right)\right. \\
& \left.+\frac{p_{a} \rho_{b}^{*} \rho_{c}^{*}}{2 \rho_{0} p_{0} C_{s}^{2}}+\frac{\rho_{a} \theta_{b}^{*} \theta_{c}^{*}}{2 \rho_{0}^{2} N^{2}}-\frac{\theta_{a} * \theta_{b}^{*} \theta_{c}^{*}}{3 N^{2} \rho_{0}^{2} g}-\frac{4+\kappa}{6 \rho_{0} C_{s}^{2} p_{0}} p_{a} p_{b}^{*} p_{c}^{*}+\mathrm{CP}\right\} d x d y d z
\end{aligned}
$$


where CP means the same term as what is preceding but with cyclic permutations among the subscripts $a b c$. On the other hand, from equation (4.3) it follows

$$
\begin{aligned}
E_{a} \frac{d\left|A_{a}\right|^{2}}{d t} & =\sum_{b} \sum_{c} \sigma_{a}^{b c} \operatorname{Im}\left(A_{a}^{*} A_{b} A_{c} e^{-i \delta_{a b c} t}\right) \\
\frac{d\left(A_{a} A_{b}^{*} A_{c}^{*}\right)}{d t} & =\mathcal{O}\left(A^{4}\right)
\end{aligned}
$$

Taking the time-derivative of (4.9a), (4.9b) and using (4.12) we get

$$
\sum_{a} \sum_{b} \sum_{c}\left[\sigma_{a}^{b c}+S_{a b c} \delta_{a b c}\right] 2 i \operatorname{Im}\left(A_{a}^{*} A_{b} A_{c} e^{-i \delta_{a b c} t}\right)+\mathcal{O}\left(A^{4}\right)=0
$$

Equation above shows that total pseudoenergy is no longer conserved for an arbitrary truncation of $(4.1)^{1}$. Nevertheless, the rate of change of total pseudoenergy for an arbitrary truncation of our model equations is of $\mathcal{O}\left(|A|^{4}\right)$. Thus, for small disturbances, the variation of total pseudoenergy will also be small, even for truncated models. Conversely, regardless of the modal truncation, a necessary condition for equation (4.13) to hold is that the sum inside the square brackets must vanish identically for all interacting triads, namely

$$
\sigma_{a}^{b c}-\sigma_{b}^{a c}-\sigma_{c}^{a b}=-\delta_{a b c} S_{a b c}
$$

For resonant triads $\left(\delta_{a b c}=0\right)$ or triads containing only vortical modes (i.e. modes with $\omega \equiv 0)$, the constraint above reduces to

$$
\sigma_{a}^{b c}-\sigma_{b}^{a c}-\sigma_{c}^{a b}=0
$$

For these kinds of triads, the quadratic component $\mathcal{A}^{(2)}$ of total pseudoenergy is exactly conserved. Consequently, the wave mode with the largest absolute value coupling constant of the triad (say, mode $a$ ) will always receive energy from or lose to the remaining triad components. Moreover, in these resonant interactions, condition $\sigma_{a}^{b c}-\sigma_{b}^{a c}-\sigma_{c}^{a b}=0$, together with the resonance relation $\omega_{a}-\omega_{b}-\omega_{c}=0$, implies that this mode having the largest absolute value coupling coefficient will always be the one with the largest absolute eigenfrequency. This mode is usually labeled as pump wave from the context of plasma physics (Weiland and Wilhelmsson, 1977). For brevity in exposition, henceforth we will keep using this notation when referring to this mode.

\footnotetext{
${ }^{1}$ Actually, total pseudoenergy of model equations (3.45) is not exactly conserved even for the full expansion case due to the $\mathcal{O}\left(u^{\prime}, v^{\prime}, w^{\prime}, p^{\prime}, \theta^{\prime}\right)^{4}$ terms in (3.31).
} 
In contrast, equation (4.14) implies that any non-resonant interacting triplet involving acoustic and/or gravity waves undergoes time variations of $\mathscr{A}^{(2)}$, which occur partially at the expense of time variations of $\mathscr{A}^{(3)}$ associated with the referred triad. Table 2 shows a sample of interacting triads involving acoustic and gravity wave types. It can be noted that, in general, even for triads involving two gravity modes and one acoustic mode, which are characterized by a large frequency mismatch $\delta_{a b c}$, the acoustic mode is always the pump mode in this kind of triad interaction. Nevertheless, the large time-frequency mismatch associated with this triad interaction type inhibits the energy exchanges among the mode components since it requires an unrealistically high amplitude of the acoustic mode in order for pump wave instability to occur, as will be shown in the next section.

\subsection{Analysis of Highly Truncated Spectral Solutions}

Given the general theoretical framework of the nonlinear interaction among the wave modes of our nonhydrostatic model employed in the previous section, in this section we will further investigate highly truncated versions of the interaction equations (4.3) to analyse the possibility of acoustic modes to excite inertio-gravity waves. First we will consider the most elementary form of the interaction equations: a single interacting wave triplet. Then we augment our analysis for considering two coupled interacting wave triads.

\subsubsection{Single triad interaction}

Consider the modal expansion (4.1) for a single interacting triad of modes $(a, b, c)$, equation (4.3) now reads:

$$
\begin{aligned}
& E_{a} A_{a}^{\prime}=\sigma_{a}^{b c} A_{b} A_{c} e^{-i \delta_{a b c} t}, \\
& E_{b} A_{b}^{\prime}=\sigma_{b}^{a c} A_{a} A_{c}^{*} e^{i \delta_{a b c} t}, \\
& E_{c} A_{c}^{\prime}=\sigma_{c}^{a b} A_{a} A_{b}^{*} e^{i \delta_{a b c} t} .
\end{aligned}
$$

In the case of a resonant interaction $\left(\delta_{a b c}=0\right)$, it is well known that if one of the wave modes hold most part of the initial energy of the triplet, this mode will only be unstable if it is the pump wave of the triad (see Craik 1988, Chapter 8). Here we extend such linear stability analysis for an arbitrary value of the mismatch $\delta_{a b c}$ to encompass all the possibilities of interacting triads involving acoustic and gravity modes in the present model. 
To study the stability of one wave mode in the single-triad interaction equations above, it is suitable to make the transformation of variable $A_{b}(t)=\tilde{A}_{b}(t) e^{i \delta_{a b c} t}$ (Lopes and Chian, 1996). Inserting this transformation, equations (4.15) become

$$
\begin{aligned}
E_{a} A_{a}^{\prime} & =\sigma_{a}^{b c} A_{b} A_{c}, \\
E_{b}\left(A_{b}^{\prime}+i \delta_{a b c} A_{b}\right) & =\sigma_{b}^{a c} A_{a} A_{c}^{*}, \\
E_{c} A_{c}^{\prime} & =\sigma_{c}^{a b} A_{a} A_{b}^{*},
\end{aligned}
$$

where the " " has been omitted to avoid cumbersome notation. Notice that $\left|A_{b}(t)\right|=$ $\left|\tilde{A}_{b}(t)\right|$.Let us assume that Mode $a$ is the pump mode of the triad (i.e., $\left.\left|\sigma_{a}^{b c}\right|>\max \left(\left|\sigma_{b}^{a c}\right|,\left|\sigma_{c}^{a b}\right|\right)\right)$ and its initial energy level dominates the total energy of the triad, that is, $\left|A_{a}(0)\right| \gg$ $\max \left(\left|A_{b}(0)\right|,\left|A_{c}(0)\right|\right)$. With these assumptions, equations (4.16) can be approximated by their linearized version around Mode $a$ 's amplitude

$$
\begin{array}{r}
A_{a}(t)=A_{a}(0)=\text { const } \\
A_{b}^{\prime \prime}+i \delta_{a b c} A_{b}^{\prime}=-\frac{\sigma_{c}^{a b} \sigma_{b}^{a c}}{E_{c} E_{b}}\left|A_{a}\right|^{2} A_{b} .
\end{array}
$$

Thus, as the coupling constants are purely imaginary numbers, in order for instability to occur two conditions must be satisfied:

$$
\begin{array}{r}
\operatorname{Im}\left(\sigma_{b}^{a c}\right) \operatorname{Im}\left(\sigma_{c}^{a b}\right)>0, \text { and } \\
\delta_{a b c}^{2}<\frac{4 \operatorname{Im}\left(\sigma_{b}^{a c}\right) \operatorname{Im}\left(\sigma_{c}^{a b}\right)}{E_{b} E_{c}}\left|A_{a}\right|^{2} .
\end{array}
$$

Otherwise, the solution is stable, and no amplification of Modes $b$ and $c$ occurs. Condition (4.18a) says that Mode $a$ must be the pump mode of the triad. For an exact resonant interaction, this condition is the only requirement for instability to occur. Conversely, according to condition (4.18b), the minimal value of Mode $a$ 's amplitude for instability to occur increases linearly as the absolute value of the mismatch parameter increases.

Consequently, for an interacting wave triplet composed of two inertio-gravity waves and one inertio-acoustic mode, the amplitude regime of the pump mode required to yield instability might be so high to be observable in the real atmosphere due to the high timefrequency mismatch among the triad components in this case. For example, for the case of Triad 3 of Table 4.1 composed of mesoscale acoustic and gravity modes, the frequency 
mismatch is $\frac{\delta_{a b c}}{2 \Omega} \approx 378$, giving a threshold amplitude associated with vertical wind perturbations of the order of $200 \mathrm{~m} / \mathrm{s}$. Thus, for an acoustic mode amplitude value yielding dynamical field perturbations with realistic values, there is no pump wave instability for this triplet. Fig. 4.2 displays the result of a time integration of the full nonlinear version of the three-wave interaction equations (4.15) for an amplitude value of the pump mode of Triad 3 of Table 4.1 that yields realistic values of the perturbation field variables. This figure confirms that, for the mode amplitudes associated with realistic values of atmospheric flow disturbances, the high frequency mismatch associated with the interacting triads involving an acoustic mode and two gravity waves strongly inhibits the energy exchanges among the modes of such triads.

In contrast, an acoustic-inertia wave mode can undergo pump wave instability in near resonant triads involving another acoustic-inertia wave and an inertio-gravity mode. In fact, for the parameters of Triad 1 of Table 4.1 and the value of $\left|A_{a}\right|$ chosen to yield a vertical wind magnitude of $\approx 2.4 \mathrm{~m} / \mathrm{s}$, parametric instability does occur. Numerical integration of the full equations (4.15) in this referred amplitude regime illustrates the expressive energy modulations undergone by the three wave modes (Fig. 4.1) of this triplet.

Teruya (2014) analysed the dynamics of a single resonant triad interaction with a wave triplet similar to the one illustrated in Fig. 4.1. They analysed the analytical solution of the interaction equations (4.15) for the exact resonant case $\delta_{a b c}=0$ with $\left|A_{a}(t=0)=0\right|$ and discussed the consequences of the mode energy modulations for the physical space solution $\left[u^{\prime}, v^{\prime}, w^{\prime}, p^{\prime}, \theta^{\prime}\right]$ in view of the energy partition of each mode type on the kinetic, available elastic and available potential forms. As in the full system (4.3) a single wave mode may participate of several connected wave triplets, to investigate in a simplified fashion the possibility of each of the wave modes excited by the pump acoustic wave instability illustrated in Fig. 4.1 to excite other gravity wave modes, we shall augment our analysis of the phase-space dynamics to consider two triads coupled by a single mode. 


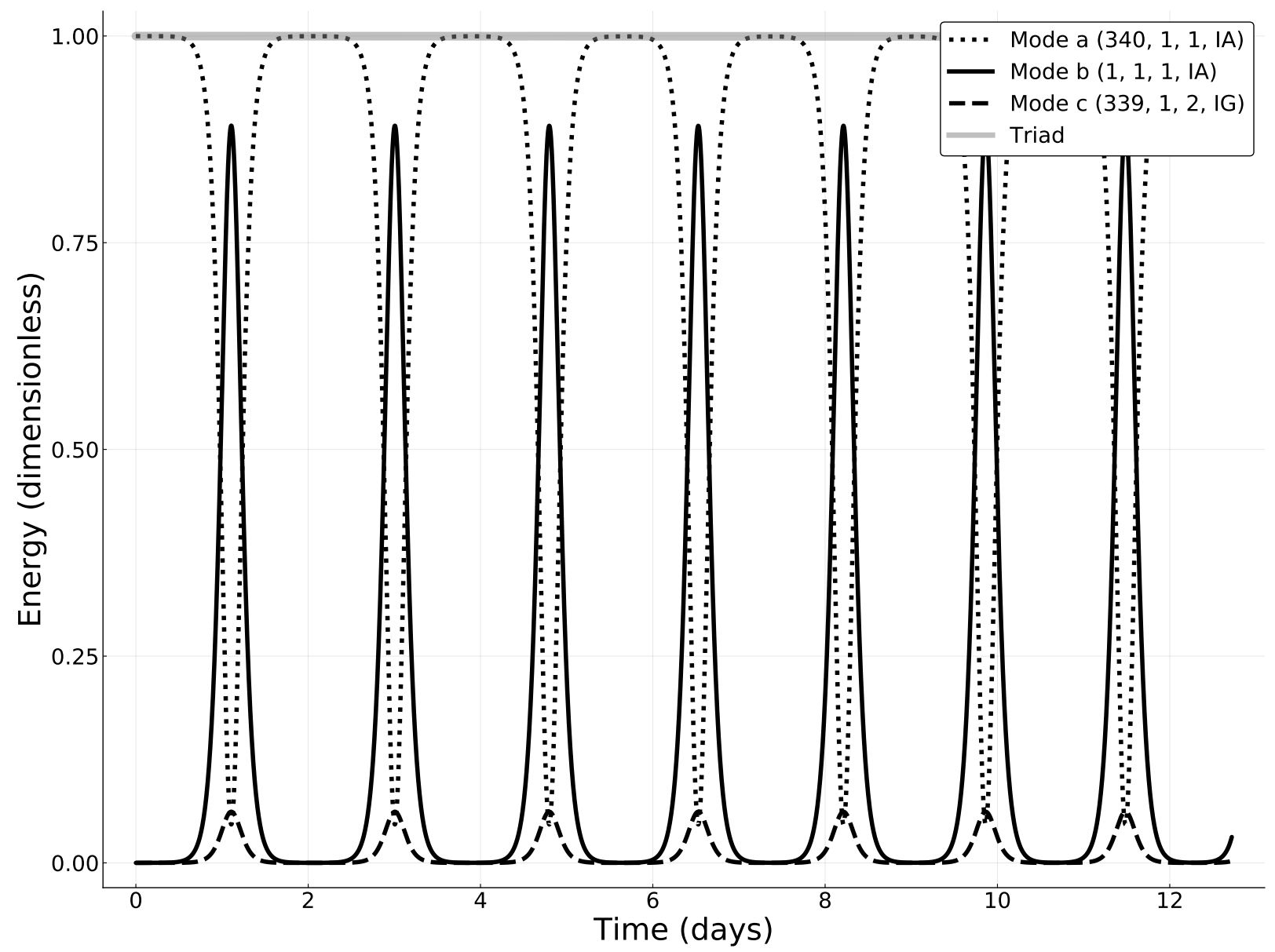

Figure 4.1: Time evolution of the energy of a resonant triad (triad 1 from Table 4.1).

\subsubsection{Two triads coupled by one mode}

Let us now consider a truncated version of modal expansion (4.1) that considers five modes $(a, b, c, d, e)$ whose wavenumbers and eigenfrequencies satisfy the relations

$$
\begin{aligned}
& m_{a}=m_{b}+m_{c}, \\
& n_{a}=n_{b}+n_{c}, \\
& k_{a}= \pm k_{b} \pm k_{c} \\
& \omega_{a}=\omega_{b}+\omega_{c}+\delta_{a b c}, \\
& m_{c}=m_{d}+m_{e}, \\
& n_{c}=n_{d}+n_{e}, \\
& \omega_{c}=\omega_{d}+\omega_{e}+\delta_{c d e} .
\end{aligned}
$$

Notice that we have imposed the interaction condition for the vertical wavenumbers only to the primary triad $(a, b, c)$. This is because the condition for the vertical wavenumbers is 


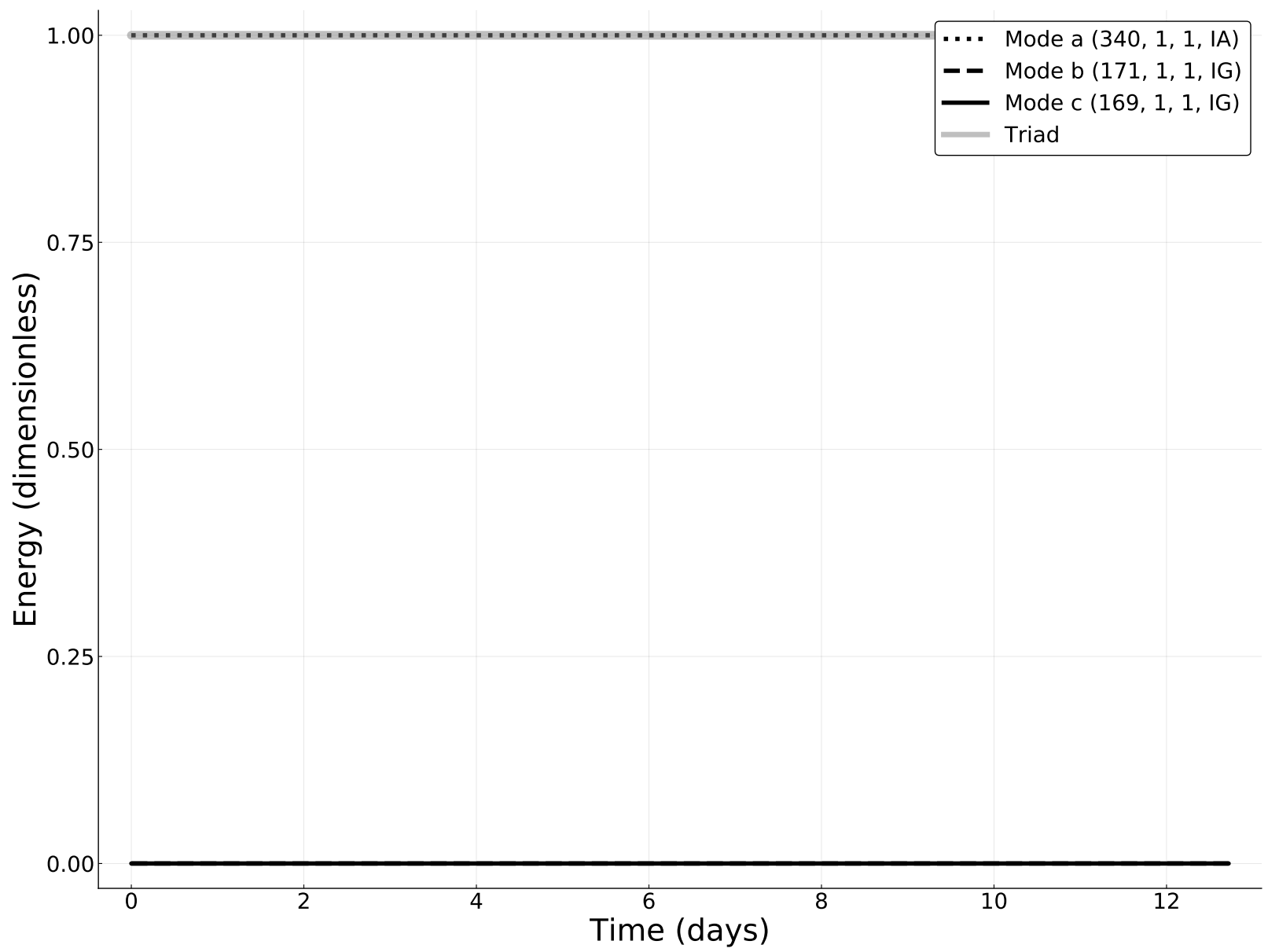

Figure 4.2: Time evolution of the energy of a nonresonant triad (triad 14 from Table 4.1).

no longer excluding. So, other triads that do not satisfy $k_{c}= \pm k_{d} \pm k_{e}$ can be excited by the mode coupling the two triads. In this situation, equation (4.3) now reads

$$
\begin{aligned}
E_{a} A_{a}^{\prime} & =\sigma_{a}^{b c} A_{b} A_{c} e^{-i \delta_{a b c} t} \\
E_{b} A_{b}^{\prime} & =\sigma_{b}^{a c} A_{a} A_{c}^{*} e^{i \delta_{a b c} t}, \\
E_{c} A_{c}^{\prime} & =\sigma_{c}^{a b} A_{a} A_{b}^{*} e^{i \delta_{a b c} t}+\sigma_{c}^{d e} A_{d} A_{e} e^{-i \delta_{c d e} t}, \\
E_{d} A_{d}^{\prime} & =\sigma_{d}^{c e} A_{c} A_{e}^{*} e^{i \delta_{c d e} t} \\
E_{e} A_{e}^{\prime} & =\sigma_{e}^{c d} A_{c} A_{d}^{*} e^{i \delta_{c d e} t}
\end{aligned}
$$

In order to analyze the stability of the triad interaction $(a, b, c)$ to small amplitude perturbations associated with the interacting modes $(c, d, e)$, let us assume first that $\mid A_{c}(t=$ $0) \mid \gg \max \left(\left|A_{d}(t=0)\right|,\left|A_{e}(t=0)\right|\right)$. In this case, Mode $c$ evolves independently of Modes $d$ and $e$, and its amplitude obeys the three wave equations (4.15). Furthermore, by explicitly expressing $A_{c}, A_{d}$ and $A_{e}$ in terms of their real and imaginary parts, the linearized version 
of equations (4.20d)-(4.20e) around the amplitude of Mode $c$ can be written as

$$
\mathbf{X}^{\prime}=M(t) \mathbf{X}
$$

where $\mathbf{X}=\left[A_{d_{r}}, A_{d_{i}}, A_{e_{r}}, A_{e_{i}}\right]^{T}$ and the matrix $M(t)$ is defined by

$$
M(t)=\left[\begin{array}{cc}
0_{2 \times 2} & M_{d}(t) \\
M_{e}(t) & 0_{2 \times 2}
\end{array}\right]
$$

where $0_{2 \times 2}$ is the two by two zero matrix and $M_{d}(t)$ is defined as

$$
M_{d}(t)=\left[\begin{array}{cc}
-\frac{\operatorname{Im}\left(\sigma_{d}^{c e}\right)}{E_{d}}\left(A_{c_{r}} \sin \left(\delta_{c d e} t\right)+A_{c_{i}} \cos \left(\delta_{c d e} t\right)\right) & \frac{\operatorname{Im}\left(\sigma_{d}^{c e}\right)}{E_{d}}\left(-A_{c_{i}} \sin \left(\delta_{c d e} t\right)+A_{c_{r}} \cos \left(\delta_{c d e} t\right)\right) \\
\frac{\operatorname{Im}\left(\sigma_{d}^{c e}\right)}{E_{d}}\left(A_{c_{r}} \cos \left(\delta_{c d e} t\right)-A_{c_{i}} \sin \left(\delta_{c d e} t\right)\right) & \frac{\operatorname{Im}\left(\sigma_{d}^{c e}\right)}{E_{d}}\left(A_{c_{i}} \cos \left(\delta_{c d e} t\right)+A_{c_{r}} \sin \left(\delta_{c d e} t\right)\right)
\end{array}\right]
$$

If the matrix coefficient $M(t)$ is periodic, Floquet's theorem (Arnol'd, 1989; Vanneste and Vial, 1994; Majda, 2003) can be used to study the stability of system (4.21). Conversely, as we are considering arbitrary time-frequency mismatchs $\delta_{a b c}$ and $\delta_{c d e}$, in order for $M(t)$ to be exactly periodic (and, therefore, Floquet theory be applicable), two conditions must be met: i) the mismatch $\delta_{a b c}$ and the nonlinear oscillation frequency of the spectral amplitudes $A_{a}, A_{b}$ and $A_{c}$ must be co-measurable; and ii) the resulting oscillation frequency of $A_{c}(t)$ be also co-measurable with the second triad mismatch $\delta_{c d e}$. As these two conditions are very restrictive, a more general way to analyze the stability of the aforementioned linear system is to estimate its maximal Lyapunov exponent (MLE) (Zounes and Rand, 1998), for which the MLE being positive means instability. The MLE has been evaluated using the method described in Benettin et al. (1976), whose implementation is available in (Datseris, 2018).

For the 5-wave system composed of the modes of Triads 1 and 2 of Table 4.1, the MLE of system (4.21) associated with the linearized dynamics of the gravity modes $(170,1,2)$ and $(169,1,2)$ is $\Lambda_{L}=1.35 \times 10^{-5} s^{-1}$, corresponding to a growth rate of $1 / 20.5 h^{-1}$, which seems compatible with the typical time-scale of internal-gravity waves. In this case, the time modulation of $A_{c}(t)$ refers to the solution illustrated in Fig. 4.1. The instability of the gravity wave-mode $(339,1,2)$ to the other two gravity modes of Triad 2 of Table 4.1 is illustrated in the numerical integration of system (4.21) displayed in Fig. 4.3, which shows the growth of the gravity wave harmonics $(170,1,2)$ and $(169,1,2)$. Since the gravity mode $(339,1,2)$ representing Mode $\mathrm{c}$ in this example is the pump wave of the triplet $(c, d, e)$, 
this instability appears more likely to be a pump wave instability than a modulational instability explored by Connaughton et al. (2010) in the Rossby wave context.

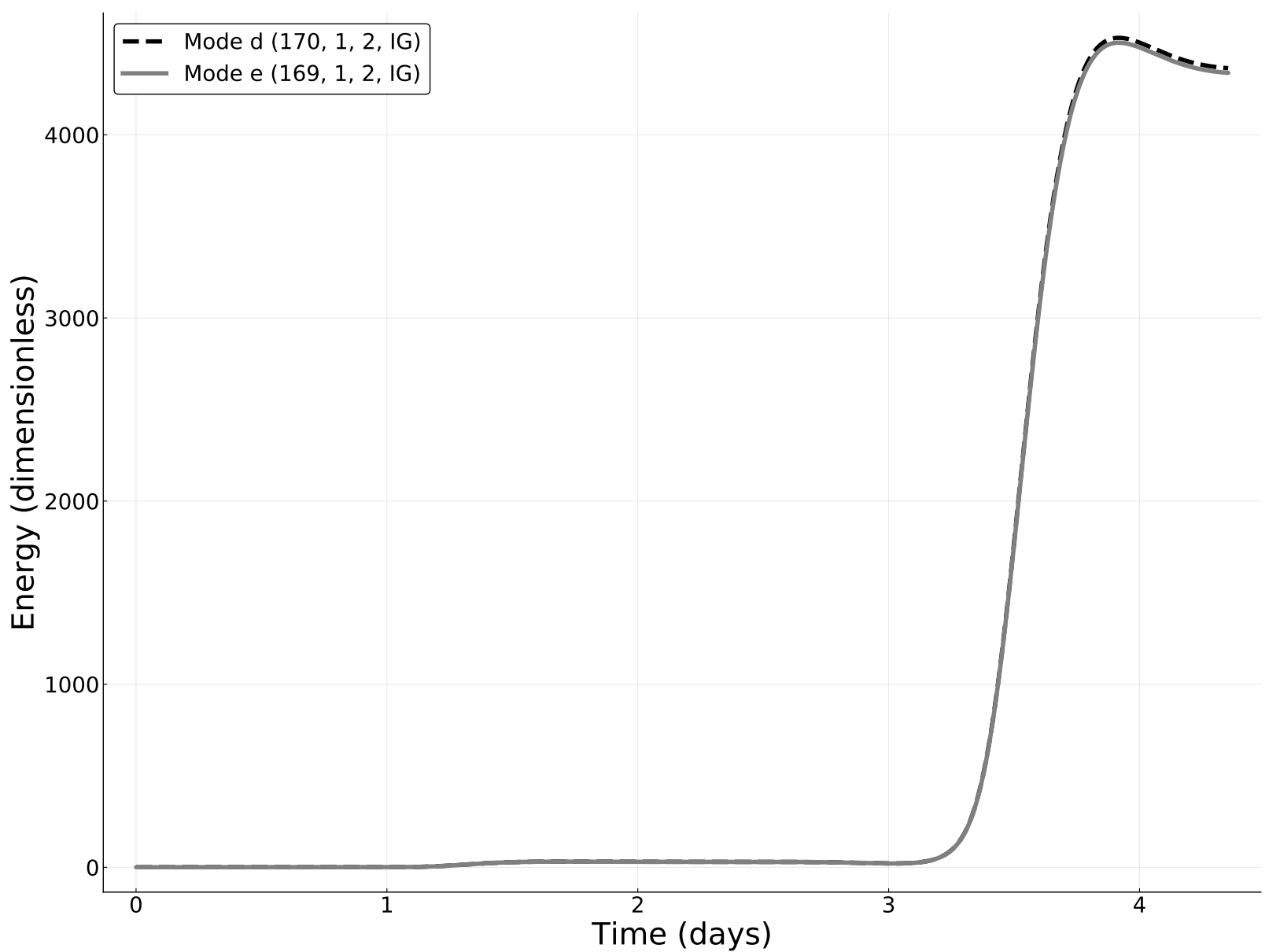

Figure 4.3: Numerical solution of the duet system forced by triad 1 and composed by modes $(170,1,2$, IG), $(169,1,2, \mathrm{IG})$ from triad 2. This solution presented a maximal Lyapunov exponent $\lambda_{L}=1.35 E-5$.

In fact, if we consider the coupling between Triads 1 and 13 of Table 4.1 through the same gravity mode $(339,1,2)$, that is, if we replace Triad 2 to Triad 13 (which has a larger time-frequency mismatch) in our 5-wave system, the solution of (4.21) is stable $\left(\operatorname{MLE} \Lambda_{L}=-4.85 \times 10^{-10} s^{-1}\right)$ and no amplification of a pair of other gravity waves occurs (Fig. 4.4). Fig. 4.5 illustrates a similar situation when Modes $d$ and $e$ are gravity modes and Mode $c$ is an inertio-acoustic mode. In this case, the planetary acoustic mode of Triad 1 of Table 4.1 couples this triad with the two inertio-gravity modes of Triad 15, and the MLE of system (4.21) is positive but very close to zero. In fact, the MLE in this case is $\Lambda_{L}=6.59 \times 10^{-11} \mathrm{~s}^{-1}$, corresponding to a growth rate of $\approx 1 / 180 \mathrm{day}^{-1}$, which is no longer compatible with the observed time-scale of gravity waves. Therefore, in this situation, as in the previous case displayed in Fig. 4.4, system (4.21) is stable and there is no amplification of the two gravity modes. The numerical integration of the nonlinear 
five-wave system (4.20) for the corresponding stable cases of the linearized system (4.21) displayed in Figs. 4.4 and 4.5 confirms that there is no energy leakage from Triad 1 towards the other gravity modes, so that the time evolution of Modes $(a, b, c)$ is identical to that predicted by the three-wave problem displayed in Fig. 4.1 (figures not shown).

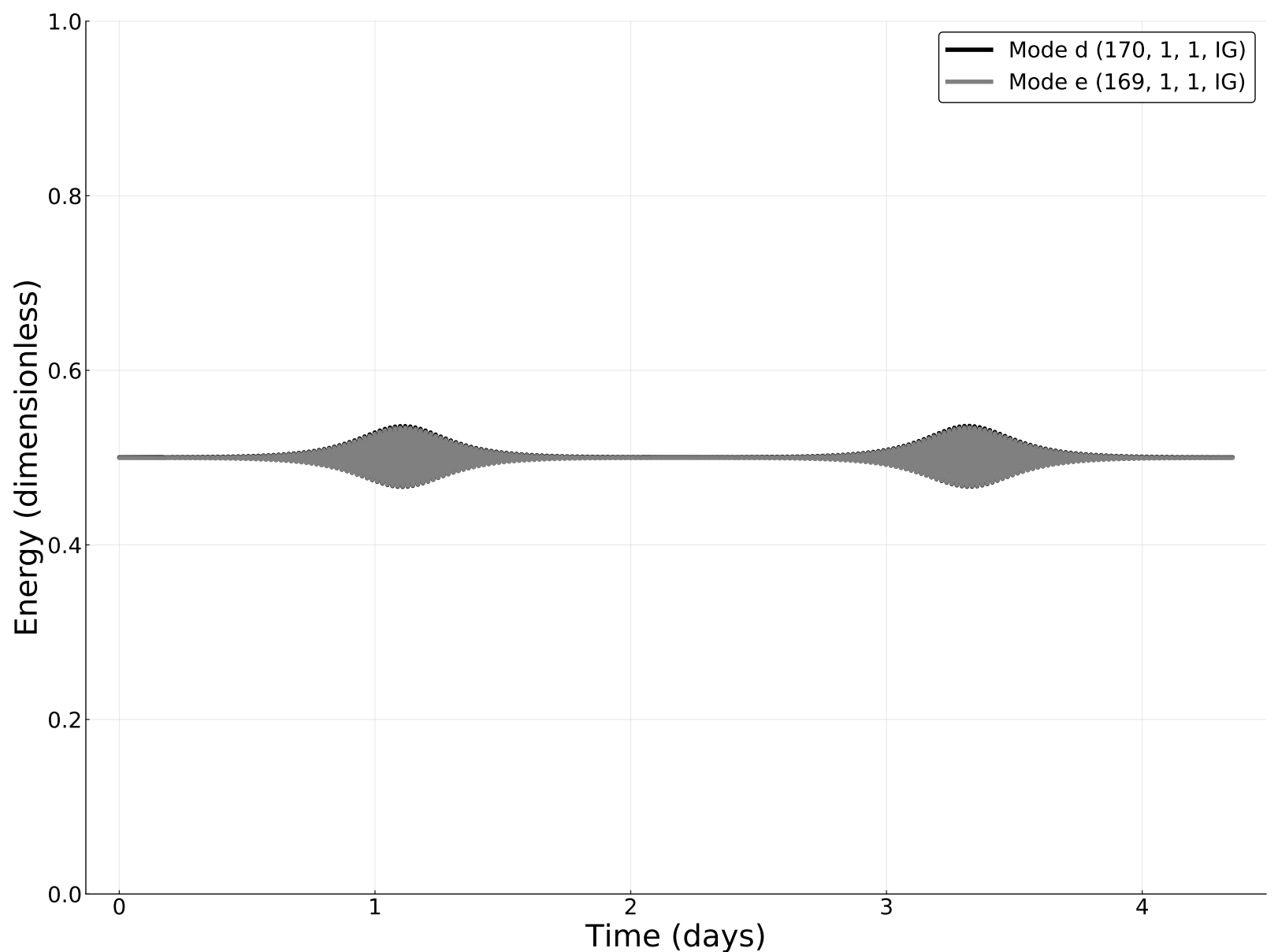

Figure 4.4: Numerical solution of the duet system forced by triad 1 and composed by modes (170,1,1,IG), $(169,1,1, \mathrm{IG})$ from triad 13. This solution presented a maximal Lyapunov exponent $\lambda_{L}=-4.85 E-10$.

A result of numerical integration of the full 5-wave system for the unstable case of system (4.21) is displayed in Figs. 4.6 and 4.7. In this numerical integration, the initial condition is similar to those considered in Fig. 4.1, in which the pump acoustic wave (340, $1,1)$ holds almost all initial energy of the system, with only a small energy perturbation distributed among the remaining modes $b, c, d$ and $e$. In this experiment Mode $a$ excites Modes $b$ and $c$ through pump wave instability (Figs. 4.6). After that, part of Mode $c$ ' energy leaks to the gravity modes $(170,1,2)$ and $(169,1,2)$ (Modes $d$ and $e$ ), yielding the excitation of these modes (Fig. 4.7). In this case, Mode $c$ acts as the pump mode in the triad interaction with Modes $d$ and $e$. Consequently, the time evolution of the mode energies of the five-wave system in this case shows that, apart from the considerable 


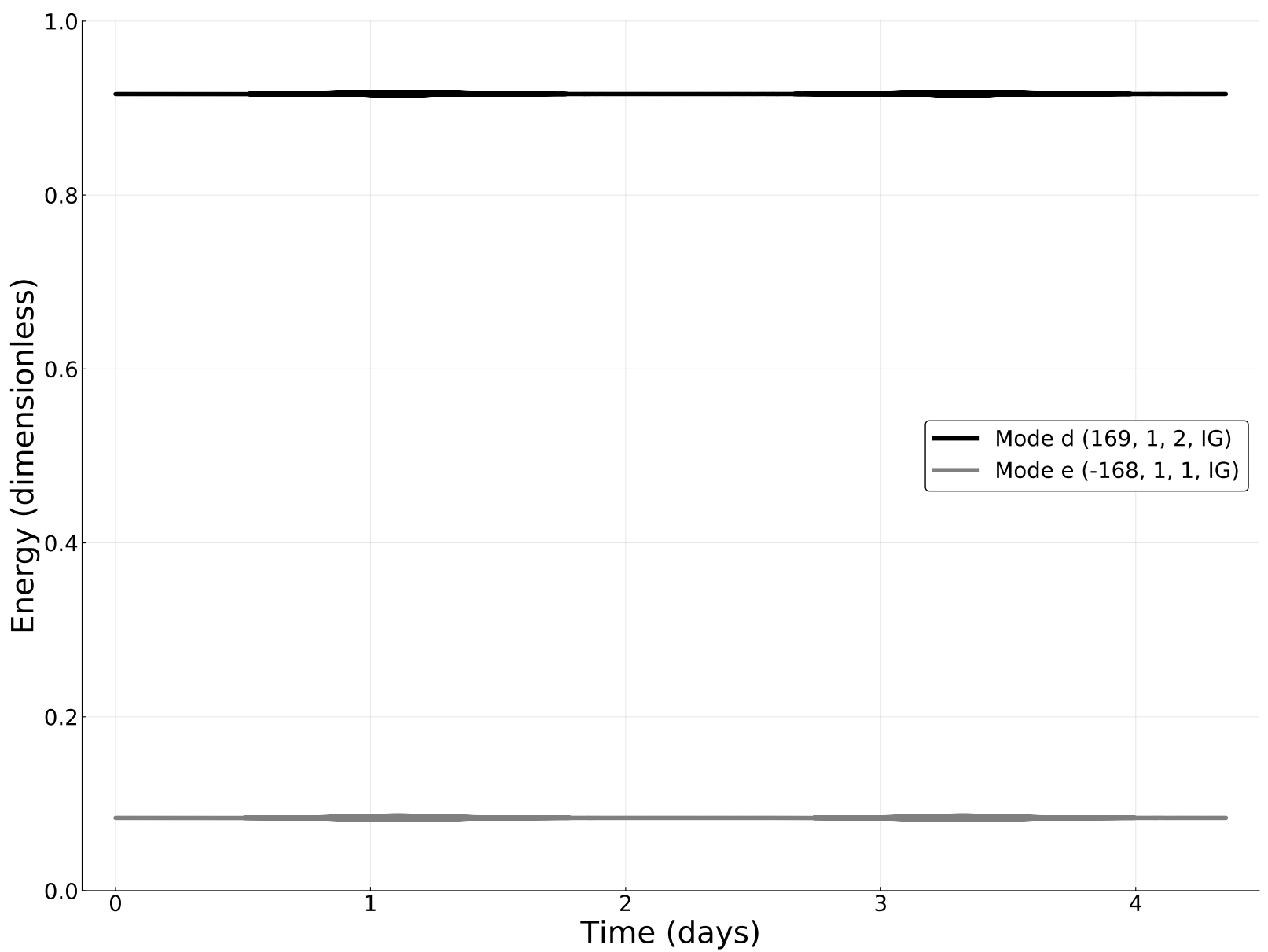

Figure 4.5: Numerical solution of the duet system forced by triad 1 and composed by modes $(169,1,2, \mathrm{IG})$, $(-168,1,1, \mathrm{IG})$ from triad 15 . This solution presented a maximal Lyapunov exponent $\lambda_{L}=6.59 E-11$.

energy modulations of the gravity modes $d$ and $e$ at the expense of Mode $c$, the coupling of Mode $c$ with the two gravity modes $d$ and $e$ also yields a multiplication of the periods associated with the time evolution of the energies of Modes a and b, disturbing the energy modulations of these modes from their exactly periodic nature predicted by the three wave equations (4.15).

Another interesting feature regarding the unstable case is that the eigenfrequencies of the gravity modes $(170,1,2)$ and $(169,1,2)$ representing Modes $d$ and $e$ in this example are such that the linear wave dynamics of these modes is well described by hydrostatic approximation, as well as the nonlinear interaction involving these inertio-gravity waves and vortical modes. As the nonlinear interaction involving inertio-gravity waves and vortical modes have been demonstrated to play an important role in the nonlinear geostrophic adjustment (Majda and Embid, 1998; Vanneste and Yavneh, 2004; Vanneste, 2004), the theoretical results described here suggest that acoustic modes might be important for both hydrostatic and geostrophic adjustment processes in the atmosphere. 
Table 4.1 - Each mode is represented by a zonal wavenumber, meridional wavenumber, vertical wavenumber and kind of wave. Frequencies $\omega$ and mismatch $\delta_{a b c}$ are measured in Hertz. Modes 1,2,4,9,10 and 11 are nearly resonant.

\begin{tabular}{|c|c|c|c|c|c|c|c|c|c|c|}
\hline Triad & Mode a & Mode b & Mode c & $\omega_{a}$ & $\omega_{b}$ & $\omega_{c}$ & $\delta_{a b c}$ & $i \sigma_{a}$ & $i \sigma_{b}$ & $i \sigma_{c}$ \\
\hline 1 & $(1,1,1, \mathrm{IA})$ & $(339,1,2, \mathrm{IG})$ & $(340,1,1, \mathrm{IA})$ & 5.89E-02 & $4.10 \mathrm{E}-03$ & $6.30 \mathrm{E}-02$ & 3.31E-05 & $-3.11 \mathrm{E}-01$ & $-2.16 \mathrm{E}-02$ & $-3.33 \mathrm{E}-01$ \\
\hline 2 & $(170,1,2, I G)$ & $(169,1,2, \mathrm{IG})$ & $(339,1,2, \mathrm{IG})$ & 2.09E-03 & $2.08 \mathrm{E}-03$ & $4.10 \mathrm{E}-03$ & $-6.94 \mathrm{E}-05$ & $-3.37 \mathrm{E}+02$ & $-3.35 \mathrm{E}+02$ & $-6.73 \mathrm{E}+02$ \\
\hline 3 & $(169,1,1, \mathrm{IG})$ & $(171,1,1, \mathrm{IG})$ & $(340,1,1, \mathrm{IA})$ & $3.89 \mathrm{E}-03$ & $3.93 \mathrm{E}-03$ & 6.30E-02 & $5.52 \mathrm{E}-02$ & $4.47 \mathrm{E}-01$ & $4.45 \mathrm{E}-01$ & $2.61 \mathrm{E}+00$ \\
\hline 4 & $(1,1,2, \mathrm{IA})$ & $(6,1,1, \mathrm{IA})$ & $(7,1,3, \mathrm{IA})$ & $1.11 \mathrm{E}-01$ & $5.89 \mathrm{E}-02$ & $1.65 \mathrm{E}-01$ & $-4.95 \mathrm{E}-03$ & $-2.53 \mathrm{E}-03$ & $-1.33 \mathrm{E}-03$ & $-3.80 \mathrm{E}-03$ \\
\hline 5 & $(2,1,1, \mathrm{IG})$ & $(5,1,1, \mathrm{IG})$ & $(7,1,3, \mathrm{IA})$ & $1.18 \mathrm{E}-04$ & $1.59 \mathrm{E}-04$ & $1.65 \mathrm{E}-01$ & $1.65 \mathrm{E}-01$ & $-1.55 \mathrm{E}+00$ & $-1.55 \mathrm{E}+00$ & $-2.38 \mathrm{E}-04$ \\
\hline 6 & $(10,1,1, \mathrm{IG})$ & $(329,1,2, \mathrm{IG})$ & $(339,1,1, \mathrm{IA})$ & $2.58 \mathrm{E}-04$ & 3.99E-03 & 6.30E-02 & $5.87 \mathrm{E}-02$ & $4.78 \mathrm{E}+01$ & $-5.50 \mathrm{E}+01$ & $-5.27 \mathrm{E}+00$ \\
\hline 7 & $(2,1,1, \mathrm{IA})$ & $(338,1,2, \mathrm{IG})$ & $(340,1,1, \mathrm{IG})$ & $5.89 \mathrm{E}-02$ & $4.09 \mathrm{E}-03$ & $7.43 \mathrm{E}-03$ & $-5.55 \mathrm{E}-02$ & $-1.90 \mathrm{E}+00$ & $2.73 \mathrm{E}+01$ & $2.58 \mathrm{E}+01$ \\
\hline 8 & $(5,1,1, \mathrm{IG})$ & $(350,1,2, \mathrm{IG})$ & $(355,1,1, \mathrm{IA})$ & $1.59 \mathrm{E}-04$ & $4.23 \mathrm{E}-03$ & $6.34 \mathrm{E}-02$ & $5.90 \mathrm{E}-02$ & $4.53 \mathrm{E}+01$ & $-5.06 \mathrm{E}+01$ & $-5.22 \mathrm{E}+00$ \\
\hline 9 & $(62,1,1, \mathrm{IA})$ & $(107,1,3, \mathrm{IG})$ & $(169,1,1, \mathrm{IA})$ & $5.90 \mathrm{E}-02$ & 8.96E-04 & $5.99 \mathrm{E}-02$ & $5.09 \mathrm{E}-09$ & $-4.35 \mathrm{E}-01$ & $-6.61 \mathrm{E}-03$ & $-4.42 \mathrm{E}-01$ \\
\hline 10 & $(93,1,1, \mathrm{IA})$ & $(421,1,1, \mathrm{IG})$ & $(514,1,1, \mathrm{IA})$ & $5.92 \mathrm{E}-02$ & $8.90 \mathrm{E}-03$ & $6.81 \mathrm{E}-02$ & $-5.08 \mathrm{E}-08$ & $1.06 \mathrm{E}+00$ & $1.60 \mathrm{E}-01$ & $1.22 \mathrm{E}+00$ \\
\hline 11 & $(180,1,3, \mathrm{IA})$ & $(478,1,2, \mathrm{IG})$ & $(658,1,3, \mathrm{IA})$ & $1.66 \mathrm{E}-01$ & $5.67 \mathrm{E}-03$ & $1.72 \mathrm{E}-01$ & $3.36 \mathrm{E}-08$ & $-1.74 \mathrm{E}+00$ & $-5.94 \mathrm{E}-02$ & $-1.80 \mathrm{E}+00$ \\
\hline 12 & $(87,1,1, \mathrm{IG})$ & $(252,1,3, \mathrm{IG})$ & $(339,1,2, \mathrm{IG})$ & 2.03E-03 & 2.09E-03 & 4.10E-03 & $-1.61 \mathrm{E}-05$ & $-1.53 \mathrm{E}+03$ & $-1.60 \mathrm{E}+03$ & $-3.13 \mathrm{E}+03$ \\
\hline 13 & $(170,1,1, I G)$ & $(169,1,1, \mathrm{IG})$ & $(339,1,2, \mathrm{IG})$ & 3.91E-03 & $3.89 \mathrm{E}-03$ & $4.10 \mathrm{E}-03$ & $-3.69 \mathrm{E}-03$ & $-4.82 \mathrm{E}+01$ & $-4.64 \mathrm{E}+01$ & $-9.50 \mathrm{E}+01$ \\
\hline 14 & $(1,1,1, \mathrm{IA})$ & $(339,1,1, \mathrm{IG})$ & $(340,1,1, \mathrm{IG})$ & $5.89 \mathrm{E}-02$ & 7.41E-03 & 7.43E-03 & $-5.89 \mathrm{E}-02$ & $-4.32 \mathrm{E}-01$ & $-8.10 \mathrm{E}-01$ & $-1.83 \mathrm{E}+00$ \\
\hline
\end{tabular}

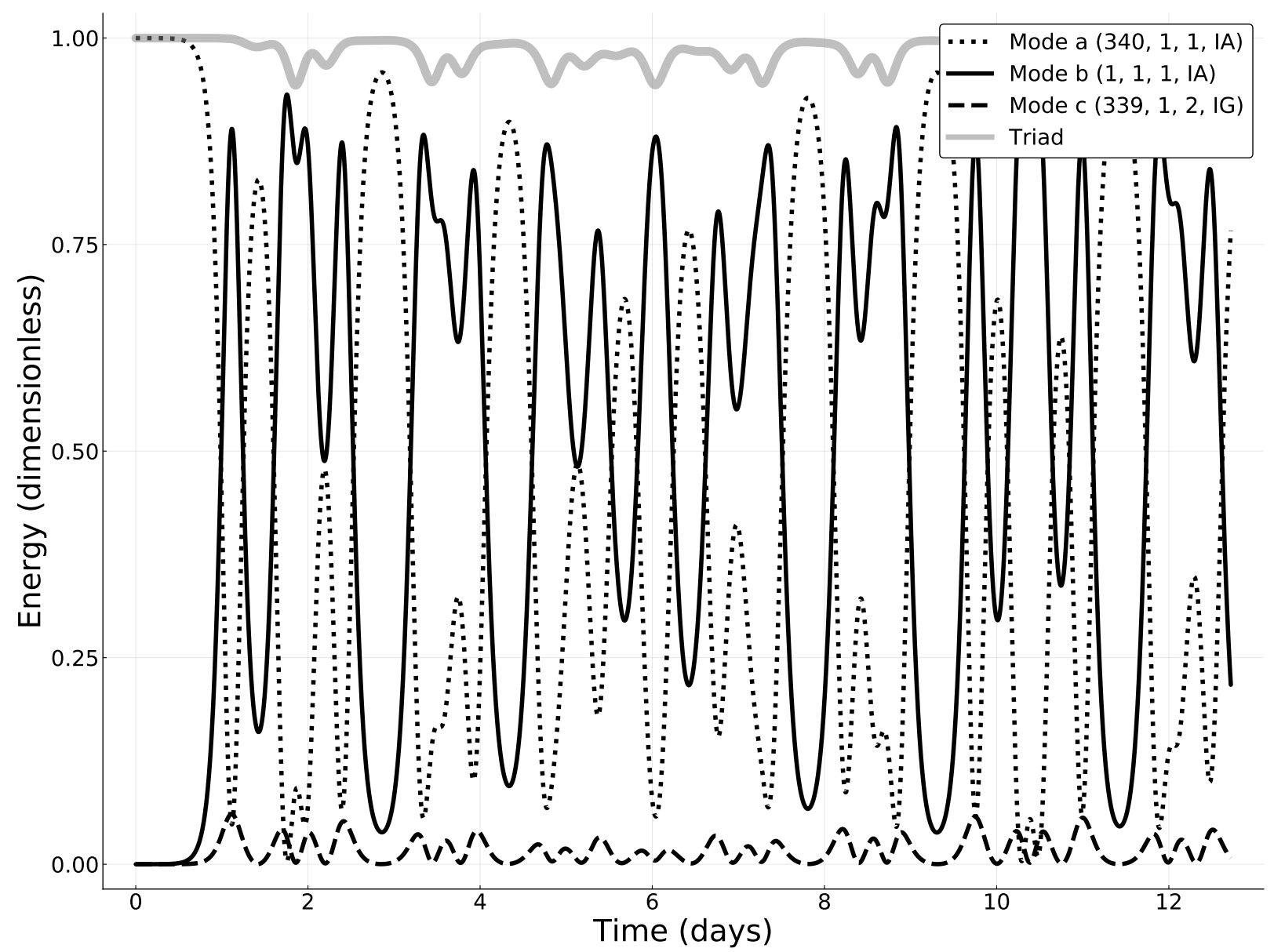

Figure 4.6: Numerical solution of a quintet composed of triads 1 and 2 from table 4.1. 


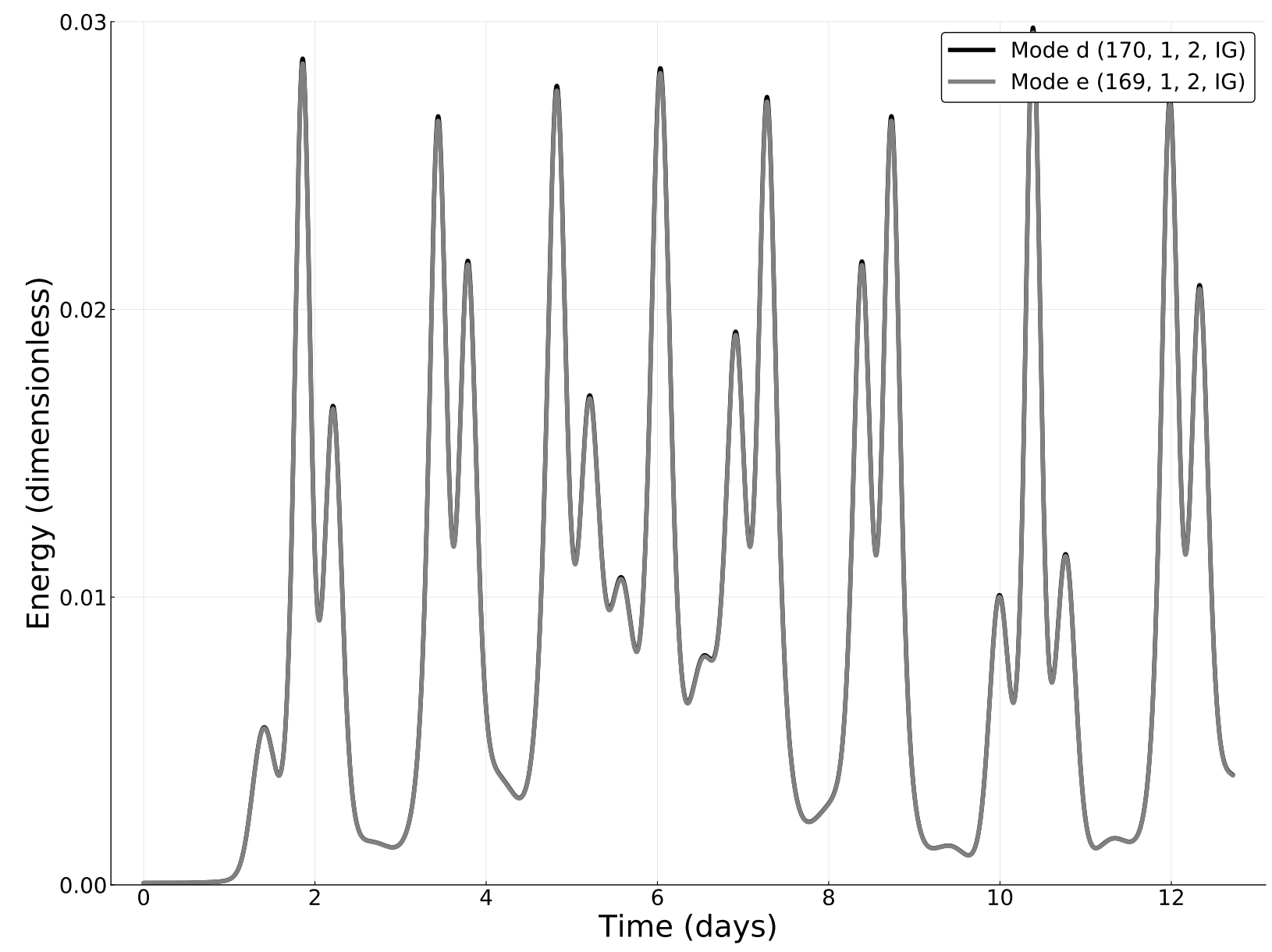

Figure 4.7: Numerical solution of a quintet composed of triads 1 and 2 from table 4.1. 
Chapter 5

\section{Conclusions}

Nonlinear triad interactions involving inertio-acoustic and inertio-gravity waves are studied here in the context of the mid-latitude $f$-plane shallow nonhydrostatic equations for a background state at rest and characterized by a hydrostatic balance and an isothermal temperature profile. In this context, we have adopted highly truncated Galerkin expansions in terms of the eigensolutions of the linear problem. For a single triad interaction, we have shown that the interacting triplets involving two inertio-gravity waves and one acoustic mode require a likely unrealistic modal amplitude regime in order for pump wave instability to occur. Consequently, for direct triad interactions, an inertio-acoustic wave mode can only be unstable to perturbations associated with a pair of acoustic/gravity modes.

In contrast, the analysis of the dynamics of two triads coupled by a single wave mode shows that a nonhydrostatic inertio-gravity wave mode (i.e., having an eigenfrequency such that the nonhydrostatic effect of vertical acceleration is not negligible) participating of a nearly resonant interaction with two acoustic modes is unstable in nearly resonant triad interactions with a pair of lower frequency inertio-gravity waves. In fact, for the representative example illustrated here in which the eigenfrequencies of the two excited inertio-gravity waves are nearly a half of the time-frequency of the primary wave (which couples the two triads), the numerical results of the nonlinear dynamics of the five-wave system confirm that this instability yields energy modulations on the two secondary gravity modes. Since the higher the time frequency the more important the nonhydrostatic effect of vertical acceleration on the inertio-gravity waves, the results suggest that inertio-acoustic waves may induce hydrostatically balanced inertio-gravity waves to undergo episodic amplitude (energy) modulations due to inter-triad energy exchanges. On the other hand, if one of the acoustic modes of a resonant interaction involving acoustic/gravity waves couple the 
two triads in our reduced 5-wave dynamics, our results show that the maximum Lyapunov exponent of the corresponding linearized system for the two gravity waves gives a growth time-scale of $\approx 180$ days, which is no longer compatible with the observed time-scale of internal gravity waves and, consequently, the resultant instability might likely be irrelevant in the atmosphere.

As discussed in Chapter 1, due to the ultra-high frequency of acoustic waves, their numerical treatment using explicit schemes implies highly restrictive computational constraints. Consequently, in nonhydrostatic numerical models adopted for meso-scale simulations the acoustic modes are either filtered out or subjected to strong damping associated with implicit schemes having time-steps much higher than the acoustic cut-off period. Nonetheless, acoustic waves play an important role in the hydrostatic adjustment process as they are responsible for the vertical displacements of fluid parcels associated with the expansion of an instantaneously heated atmospheric layer (Bannon 1995, 1996; Duffy 2003). In fact, Chagnon and Bannon (2001) demonstrated that the steady-state solutions of anelastic and other sound filtering models exhibit significant differences from those of fully compressible models allowing acoustic modes.

Acoustic waves may be excited by thermal forcings associated with convective storms, especially the localized ones that have a duration shorter than the acoustic cut-off period given by $\frac{4 \pi H}{C_{S}}$ (Chagnon and Bannon 2005a,b). Our simplified theoretical model suggests that these acoustic modes generated by explosive and localized storms might play an important role in the transient phase of the three-dimensional adjustment process of the atmosphere to both hydrostatic and geostrophic balances. Specifically, this role of acoustic modes in the adjustment process of the atmosphere might be due to not only their linear energy propagation as studied by Chagnon and Bannon (2005a,b) but also their nonlinear effect of exciting hydrostatic inertio-gravity waves as pointed out by our theoretical analysis. Fanelli and Bannon (2005) investigated the hydrostatic and geostrophic adjustments to a prescribed thermal forcing utilizing a nonlinear compressible model, but they considered a heating function with a duration longer than the acoustic cut-off period of $\approx 5$ min so that no acoustic waves were excited. However, similar numerical studies with a shorter time-scale forcing should be done to apply the present theory to the hydrostatic and geostrophic adjustments in a more realistic fashion by considering the full expansion (4.1). This might be the next step in the generalization of the present theory to further 
understand both the nonlinear dynamics of the nonhydrostatic wave modes itself and its role in the nonlinear hydrostatic/geostrophic adjustment, along with testing the robustness of the present theory. 


\section{Bibliography}

Andrews D. G., A note on potential energy density in a stratified compressible fluid, J. Fluid Mech., 1981, vol. 107, p. 227

Arnol'd V., Mathematical Methods of Classical Mechanics. Graduate Texts in Mathematics, Springer, 1989

Baer F., Tribbia J., On complete filtering of gravity modes through nonlinear initialization, Mon. Wea. Rev., 1977, vol. 105, p. 1536

Bannon P. R., Hydrostatic adjustment: Lamb's problem, J. Atmos. Sci., 1995, vol. 52, p. 1743

Bannon P. R., Nonlinear hydrostatic adjustment, J. Atmos. Sci., 1996, vol. 53, p. 3606

Benettin G., Galgani L., Strelcyn J.-M., Kolmogorov entropy and numerical experiments, Phys. Rev. A, 1976, vol. 14, p. 2338

Bustamante M. D., Quinn B., Lucas D., Robust energy transfer mechanism via precession resonance in nonlinear turbulent wave systems, Phys. Rev. Letters, 2014, vol. 113, p. 084502

Chagnon J. M., Bannon P. R., Hydrostatic and geostrophic adjustment in a compressible atmosphere: Initial response and final equilibrium to an instantaneous localized heating, J. Atmos. Sci., 2001, vol. 58, p. 3776

Chagnon J. M., Bannon P. R., Wave Response during Hydrostatic and Geostrophic Adjustment. Part I: Transient Dynamics, J. Atmos. Sci., 2005a, vol. 62, p. 1311 
Chagnon J. M., Bannon P. R., Wave Response during Hydrostatic and Geostrophic Adjustment. Part II: Potential Vorticity Conservation and Energy Partitioning, J. Atmos. Sci., 2005b, vol. 62, p. 1330

Connaughton C., Nadiga B. T., Nazarenko S., Quinn B., Modulational instability of Rossby and drift waves and generation of zonal jets, J. Fluid Mech., 2010, vol. 654, p. 207

Craik A. D. D., Wave interactions and fluid flows. Cambridge Monographs on Mechanics and Applied Math. Cambridge University Press Cambridge, U.K., 1988, 322

Daley R., The normal modes of the spherical nonhydrostatic equations with applications to the filterinf og acoustic modes, Tellus, 1988, vol. 40A, p. 96

Datseris G., DynamicalSystems.jl: A Julia software library for chaos and nonlinear dynamics, Journal of Open Source Software, 2018, vol. 3, p. 598

Davies T., Staniforth A., Wood N., Thuburn J., Validity of anelastic and other equation sets as inferred from normal-mode analysis, Q. J. R. Meteorol. Soc., 2003, vol. 129, p. 2761

DeMaria M., Linear Response of a Stratified Tropical Atmosphere to Convective Forcing, J. Atmos. Sci., 1985, vol. 42, p. 1944

Domaracki A., Loesch A. Z., Nonlinear Interactions Among Equatorial Waves, J. Atmos. Sci., 1977, vol. 34, p. 486

Duffy D. G., Resonant interactions of inertio-gravity and Rossby waves, J. Atmos. Sci., 1974, vol. 31, p. 1218

Duffy D. G., Hydrostatic adjustment in nonisothermal atmospheres, J. Atmos. Sci., 2003, vol. 60 , p. 339

Fanelli P. F., Bannon P. R., Nonlinear atmospheric adjustment to thermal forcing, J. Atmos. Sci., 2005, vol. 62, p. 4253

Giraldo F. X., Restelli M., Läuter M., Semi-Implicit Formulations of the Navier-Stokes Equations: Application to Nonhydrostatic Atmospheric Modeling, SIAM J. Sci. Comput., 2010, vol. 32 , p. 3394 
Goldstein H., Classical mechanics. Addison-Wesley series in physics, Addison-Wesley Pub. Co., 1980

Greiner W., Reinhardt J., Field Quantization. Springer, 1996

Kasahara A., Normal modes of ultralong waves in the atmosphere, Mon. Wea. Rev., 1976, vol. 104 , p. 669

Kasahara A., Numerical integration of the global barotropic primitive equations with Hough harmonics expansions, J. Atmos. Sci., 1977, vol. 34, p. 687

Kasahara A., Further Studies on a Spectral Model of the Global Barotropic Primitive Equations with Hough Harmonic Expansions, J. Atmos. Sci., 1978, vol. 35, p. 2043

Kasahara A., On the Nonhydrostatic Atmospheric Models with Inclusion of the Horizontal Component of the Earth's Angular Velocity, J. Meteor. Soc. Japan, 2003a, vol. 81, p. 935

Kasahara A., The Roles of the Horizontal Component of the Earth's Angular Velocity in Nonhydrostatic Linear Models, J. Atmos. Sci., 2003b, vol. 60, p. 1085

Kasahara A., , 2004 NCAR Tech. Rep. NCAR/TN-457+STR Free oscillations of deep nonhydrostatic global atmospheres: Theory and a test of numerical schemes. NCAR Boulder, CO

Kasahara A., Gary J. M., Normal modes of an incompressible and stratified fluid model including the vertical and horizontal componentes of Coriolis force, Tellus A, 2006, vol. 58, p. 368

Kasahara A., Puri K., Spectral representation of three- dimensional global data by expansion in normal mode functions, Mon. Wea. Rev., 1981, vol. 109, p. 37

Kasahara A., Qian J. H., Normal modes of a global non-hydrostatic atmospheric model, Mon. Wea. Rev., 2000, vol. 128, p. 3357

Klein R., Asymptotics, Structure, and Integration of Sound-Proof Atmospheric Flow Equations, Theor. Comput. Fluid Dyn, 2009, vol. 23, p. 161 
Klemp J. B., Skamarock W. C., Dudhia J., Conservative Split-Explicit Time Integration Methods for the Compressible Nonhydrostatic Equations, Mon. Wea. Rev., 2007, vol. 135 , p. 2897

Klemp J. B., Skamarock W. C., Ha S., Damping Acoustic Modes in Compressible Horizontally Explicit Vertically Implicit (HEVI) and Split-Explicit Time Integration Schemes, Monthly Weather Review, 2018, vol. 146, p. 1911

Kuroda Y., On the Casimir Invariant of Hamiltonian Fluid Mechanics, J. Phys. Soc. Japan, 1990a, vol. 60, p. 727

Kuroda Y., Symmetries and Casimir Invariants for Perfect Fluid, Fluid Dyn. Res., 1990b, vol. 5 , p. 273

Landau L., Lifshitz E., Mechanics 1. Butterworth Heinemann, Elsevier ButterworthHeinemann, 1976

Leith C. E., Nonlinear Normal Mode Initialization and Quasi-Geostrophic Theory, Journal of the Atmospheric Sciences, 1980, vol. 37, p. 958

Lindzen R. D., Planetary waves on beta-planes, Mon. Wea. Rev., 1967, vol. 95, p. 441

Loesch A. Z., Deininger R. C., Dynamics of Closed Systems of Resonantly Interacting Equatorial Waves, J. Atmos. Sci., 1979, vol. 36, p. 1490

Longuet-Higgins M. S., The eigenfunctions of Laplace's tidal equation over a sphere, Philos. Tr. R. Soc. S-A., 1968, vol. 262, p. 511

Lopes S. R., Chian A. C.-L., A coherent nonlinear theory of auroral Langmuir-Alfvenwhistler (LAW) events in the planetary magnetosphere., A\&A, 1996, vol. 305, p. 669

Machenhauer B., On the dynamics of gravity oscillations in a shal- low water model, with applications to normal mode initialization, Beitr. Phys. Atmos., 1977, vol. 50, p. 253

Majda A., Introduction to Pdes and Waves for the Atmosphere and Ocean. Courant Lecture Notes in Mathematics Series, Courant Institute of Mathematical Sciences, New York University, 2003 
Majda A. J., Embid P., Averaging over fast waves for geophysical flows with unbalanced initial data, Theoretical and Computational Fluid Dynamics, 1998, vol. 11, p. 155

Marsden J., Ratiu T., Introduction to Mechanics and Symmetry. Text in Applied Mathematics, Springer, 1999

Matsuno T., Quasi-Geostrophic Motions in the Equatorial Area, J. Meteor. Soc. Japan, 1966, vol. 44, p. 25

Morrison P. J., Hamiltonian description of the ideal fluid, Rev. Mod. Phys., 1998, vol. 70, p. 467

Pedlosky J., Geophysical Fluid Dynamics. No. v. 1 in Geophysical Fluid Dynamics, Springer-Verlag, 1987, 710

Phillips N. A., Principles of large scale numerical weather prediction, Dynamic meteorology, 1973, pp 1-96

Pielke R., Mesoscale Meteorological Modeling. International geophysics series, Academic Press, 2002

Qian J. H., Kasahara A., Nonhydrostatic Atmospheric Normal Modes on Beta-Planes, Pure and Applied Geophysics, 2003, vol. 160, p. 1315

Raupp C. F. M., Silva Dias P. L., Resonant Wave Interactions in the Presence of a Diurnally Varying Heat Source, J. Atmos. Sci., 2009, vol. 66, p. 3165

Raupp C. F. M., Silva Dias P. L., Tabak E. G., Milewski P., Resonant Wave Interactions in the Equatorial Waveguide, J. Atmos. Sci., 2008, vol. 65, p. 3398

Ripa P., On the theory of nonlinear interactions among geophysical waves, J. Fluid Mech., 1981, vol. 103, p. 87

Ripa P., Weak Interactions of Equatorial Waves in a One-Layer Model. Part I: General Properties, J. Phys. Oceanogr., 1983a, vol. 13, p. 1208

Ripa P., Weak Interactions of Equatorial Waves in a One-Layer Model. Part II: Applications, J. Phys. Oceanogr., 1983b, vol. 13, p. 1227 
Ripa P., Fuentes O., Sheinbaum J., Ochoa J., Nonlinear Processes in Geophysical Fluid Dynamics: A Tribute to the Scientific Work of Pedro Ripa. Kluwer Academic Publishers, 2003

Salmon R., Lectures on Geophysical Fluid Dynamics. Oxford University Press, USA, 1998

Scinocca J. F., Shepherd T. G., Nonlinear Wave-Activity Conservarion Laws and Hamiltonian Structure for the Two-Dimensional Anelastic Equations, J. Atmos. Sci., 1992, vol. 49 , p. 339

Shepherd T. G., Symmetries, conservation lays, and Hamiltonian structure in geophysical fluid dynamics, Adv. Geophys, 1990, vol. 32, p. 287

Shepherd T. G., A unified theory of available potential energy, Atmosphere-Ocean, 1993, vol. 31 , p. 1

Silva Dias P. L., Schubert W. H., DeMaria M., Large-Scale Response of the Tropical Atmosphere to Transient Convection, J. Atmos. Sci., 1983, vol. 40, p. 2689

Taylor G. I., The oscillations of the atmosphere, Proc. Roy. Soc. London, 1936, vol. A156, p. 318

Teruya A. S. W., Energética dos Modos Normais da Dinâmica Atmosférica NãoHidrostática, São Paulo: Universidade, 2014, Master Dissertation, 92 p.

Thuburn J., , 2011 in Lauritzen P. H., Jablonowski C., Taylor M. A., Nair R. D., eds, , Vol. 80, Numerical Techniques for Global Atmospheric Models. Springer: Berlin Chapt. 1 pp 3-27

Tribbia J., Non-linear initialization on an equatorial beta-plane, Mon. Wea. Rev., 1979, vol. 107, p. 704

Vallis G. K., Atmospheric and Oceanic Fluid Dynamics. Cambridge University Press Cambridge, U.K., 2006, 745

Vanneste J., Inertia-gravity wave generation by balanced motion: revisiting the LorenzKrishnamurthy model, J. Atmos. Sci., 2004, vol. 61, p. 224 
Vanneste J., Vial F., Nonlinear wave propagation on a sphere: Interaction between Rossby waves and gravity waves; stability of the Rossby waves, Geophys. \& Astrophys. Fluid Dyn., 1994, vol. 76, p. 121

Vanneste J., Yavneh I., Exponentially Small Inertia-Gravity Waves and the Breakdown of Quasigeostrophic Balance, J. Atmos. Sci., 2004, vol. 61, p. 211

Žagar N., Gustafsson N., Källén E., Variational data assimilation in the tropics: The impact of a background-error constraint, Quart. J. Roy. Meteor. Soc., 2004, vol. 130, p. 103

Žagar N., Kasahara A., Terasaki K., Tribbia J., Tanaka H., Normal-mode function representation of global 3-D data sets: open-access software for the atmospheric research community, Geoscientific Model Development, 2015, vol. 8, p. 1169

Weiland J., Wilhelmsson H., Coherent non-linear interaction of waves in plasmas. vol. 88, Oxford Pergamon Press International Series on Natural Philosophy, 1977

White A. A., Hoskins B. J., Roulstone I., Staniforth A., Consistent approximate models of the global atmosphere: shallow, deep, hydrostatic, quasi-hydrostatic and nonhydrostatic, Q. J. R. Meteorol. Soc., 2005, vol. 131, p. 2081

Zounes R., Rand R., Transition Curves for the Quasi-Periodic Mathieu Equation, SIAM Journal on Applied Mathematics, 1998, vol. 58, p. 1094 